\title{
Electrokinetic manipulation of the von Kármán vortex street in the wake of a confined cylinder. I. DC electric field
}

Cite as: Phys. Fluids 30, 082004 (2018); https://doi.org/10.1063/1.5037595

Submitted: 25 April 2018 . Accepted: 19 July 2018 . Published Online: 08 August 2018

Dominik P. J. Barz (D), Mathias Scholz, and Steffen Hardt (iD)
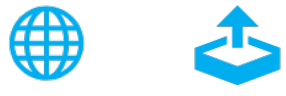

\section{ARTICLES YOU MAY BE INTERESTED IN}

Letter: An origin of magnetohydrodynamic reverse flow in $90^{\circ}$ bends

Physics of Fluids 30, 081701 (2018); https://doi.org/10.1063/1.5046328

A critical assessment of the line tension determined by the modified Young's equation

Physics of Fluids 30, 082003 (2018); https://doi.org/10.1063/1.5040574

Vibrational modes prediction for water-air bubbles trapped in circular microcavities

Physics of Fluids 30, 082001 (2018); https://doi.org/10.1063/1.5037328 


\title{
Electrokinetic manipulation of the von Kármán vortex street in the wake of a confined cylinder. I. DC electric field
}

\author{
Dominik P. J. Barz, ${ }^{1,2, a)}$ Mathias Scholz, ${ }^{1}$ and Steffen Hardt ${ }^{2}$ \\ ${ }^{1}$ Department of Chemical Engineering, Queen's University, Dupuis Hall 213, Kingston, \\ Ontario K7L 3N6, Canada \\ ${ }^{2}$ Institute for Nano- and Microfluidics, Technische Universität Darmstadt, 64287 Darmstadt, Germany
}

(Received 25 April 2018; accepted 19 July 2018; published online 8 August 2018)

\begin{abstract}
The present study is concerned with the numerical simulation of the pressure-driven flow around a confined cylinder subjected to a DC electric field. The flow situation differs from the conventional von Kármán vortex street flow in terms of confinement. Additionally, the DC electric field induces a very small but finite electrokinetic velocity at the cylinder surface in contrast to the no-slip velocity in the conventional case. Various numerical simulations are performed in the Laminar Vortex Shedding Regime to investigate the influence of the confinement and the direction and strength of the electric field. For flows without electrokinetic manipulation, the blockage ratio shifts the critical Reynolds number to higher values. Likewise, the dimensionless shedding frequency (Strouhal number) at a given Reynolds number increases with increasing blockage ratio. For flows with electrokinetic manipulation, the time that is required to obtain a steady Laminar Vortex Shedding Regime is reduced compared to the corresponding pure pressure-driven flow. Steady electrokinetic manipulation does not influence the dimensionless shedding frequency. The reduction of the transient is related to differences in the flow topologies around the cylinder. The electrokinetic velocity breaks the axial flow symmetry of the Laminar Steady Regime and therefore accelerates the onset of the Laminar Vortex Shedding Regime. Published by AIP Publishing. https://doi.org/10.1063/1.5037595
\end{abstract}

\section{INTRODUCTION}

Electrokinetic phenomena become important in fluid systems with a high surface-area-to-volume ratio such as in microfluidic devices. They arise from the interaction of an external force with an electric double layer (EDL). An EDL is formed when an electrically charged surface is in contact with a liquid containing ions. Counter- and co-ions are attracted and repelled, respectively. The excess of counter-ions violates the electric neutrality of the liquid. Hence, an application of an external electric field induces an electrostatic force in the EDL. This drives the liquid in the EDL, while the adjacent (electrically neutral) bulk liquid is dragged by viscous interactions; the so-called electroosmosis or electrokinetic flow (EKF). To induce a measurable EKF in a channel, its diameter must be roughly less than a millimeter. Hence, EKF is not only a surface-based phenomenon but also a typical microscale phenomenon. Review on electrokinetic phenomena and electrokinetic flows is available in Refs. 1-3.

The periodic separation of a flow around a bluff body is called the von Kármán Vortex Street (KVS) and can be observed on very different length scales. For example, it is found in the trails behind ocean islands such as Madeira ${ }^{4}$ and wind scrapers and chimneys ${ }^{5}$ but also on a much smaller length scale such as in the wake of nuclear fuel rods or heat exchanger pipes. ${ }^{6}$ The size (typical length scale) of the bluff body does not play a role and flows around similar geometries with the

a)Electronic mail: dominik.barz@queensu.ca same ratio of relevant forces- that is equal Reynolds numbers $R e$-share identical characteristics.

The flow topology around the bluff body can vary considerably with the Reynolds number. Here, we give a very limited overview for the flow around an unbounded cylinder based on the review of Williamson. ${ }^{7}$ Note that the ranges are approximate values. At $R e<5$, the streamlines are steady and symmetric and follow the cylinder geometry. The range of $R e$ $=5-49$ is called Laminar Steady Regime and is characterized by a steady recirculation area consisting of two symmetric vortices, placed on each side of the wake, that do not detach from the cylinder. The flow at $R e=49-194$ is called Laminar Vortex Shedding Regime (LVSR). Here, the recirculation area develops instabilities, initially from the downstream end of the attached vortex pair, whose strength and amplification grow as the Reynolds number grows. The wake oscillates periodically, and the vortex shedding is parallel to the cylinder axis; the flow can be considered as two-dimensional (2D). This oscillating flow is dominated by a single frequency (or mode), and the vortex street formation can be placed in the class of dynamic systems which exhibit Hopf bifurcations. ${ }^{8}$ At Reynolds numbers higher than around 190, first the wake and then the shear layer undergo a transition from a laminar to a turbulent regime. The present work is limited to the LVSR, and the different forms of the turbulent KVS are therefore not discussed.

The present research is motivated by the question whether a DC electric field applied to the EDL around a cylinder impacts the properties of the KVS. It is very difficult to induce a sufficiently strong electric field in the vicinity of a cylinder 
without disturbing the flow. We solve this problem by placing the cylinder in a (mini- or micro)-channel. This has several advantages: (i) required electric field strengths can be achieved by incorporation of electrodes in the channel walls and with realistic parameters; (ii) surface-area-to-volume is sufficient to induce a small but relevant EKF in the cylinder EDL that can influence the KVS. We call this electrokinetic manipulation from now on; and (iii) the persevering problem of finding correct boundary conditions for an unbounded cylinder flow is avoided.

Vortex separation from a confined cylinder is a well but mainly numerically investigated problem. Confined flows differ from their unbounded counterparts because of the shear in the incoming velocity profile, the shear induced close to the boundaries, and with the impermeability of the vortices through the walls. ${ }^{9}$ The early numerical work of Chen et al. showed that the critical Reynolds number $R e_{c r}$ for the onset of the Laminar Steady Regime increased with increasing ratio of cylinder diameter $d$ to channel height $h$, also called the blockage ratio $\varepsilon \equiv d / h$. The accompanying eigenvalue computations predicted that the critical Reynolds number of the LVSR increases with the blockage ratio up to around 0.5 and then decreases again. ${ }^{10}$ Numerical simulations for different positions of the cylinder along the channel height were performed by Zovatto and Pedrizzetti. For these asymmetric flows, a higher critical Reynolds number for the transition from Laminar Steady to LVSR is required. ${ }^{9}$ Further extensive numerical simulations of a confined cylinder flow in the range of $0.1 \leq R e \leq 280$ were performed by Sahin and Owens. ${ }^{11}$ The blockage ratio determines whether the change of critical Reynolds number corresponds to a Hopf or a pitchfork bifurcation. At increased blockage ratio, the flow topology alters significantly with vortices beginning to shed from both the cylinder and the confinement walls. Once the critical Reynolds number for the primary instability has been exceeded, the frequency at which periodic laminar vortex shedding takes place increases with increasing blockage ratio at a given Reynolds number. A non-monotonic variation of the critical Reynolds number with the blockage ratio in the LVSR is computed by Kumar and Mittal. ${ }^{12}$ It was found that as the blockage ratio increases, the critical Reynolds number for the onset of the instability first decreases and then increases. By contrast, a monotonic increase of the critical shedding frequency with increasing blockage ratio was observed. There is very limited experimental work on the topic. Rehimi et al. measured the flow field around a confined cylinder with a blockage ratio of $\varepsilon=1 / 3$ using Particle Image Velocimetry. Their results experimentally confirmed that high blockage ratios change the topology of the LVSR. Vortices that are shed from the cylinder interact with the wall boundary layer and induce another pair of (counter-rotating) vortices. ${ }^{13}$ In this regard, Griffith et al. ${ }^{14}$ identified an additional rather similar vortex shedding frequency mode at $R e=300$ and $\varepsilon=0.5$ in their numerical results. They assume that the second frequency mode is due to the wall beating, i.e., shedding of wall vortices. However, these authors performed 2D numerical computations while $R e$ $=300$ is clearly in the transitional (3D) flow regime.

In terms of electrokinetic manipulation of a KVS, we are not aware of any relevant work in the literature but the numerical study of Meisel and Ehrhard. ${ }^{15}$ In their work, an oscillating electrokinetic flow is induced to generate an artificial vortex street behind a cylinder. The study was conducted in the Laminar Steady Regime at $R e=10$, where the electrokinetic flow generates the periodic detachment of the (steady) vortex pair. By contrast, the present work is carried out in the LVSR at $50 \lesssim R e \leq 180$. Then, the KVS is a natural feature of the pressure-driven flow and we investigate the influence of a small and steady (electrokinetic) flow perturbation. In detail, we perform simulations of a flow around a confined cylinder for various Reynolds numbers and different blockage ratios with and without electrokinetic manipulations.

This article is organized as follows. First, the problem is described and mathematically formulated. Second, we present simulations concerned with the influence of the blockage ratio on the vortex shedding of a pure pressure-driven flow. These results are compared with literature data to validate our methodologies. Third, we present simulations with different electrokinetic manipulations and analyze the influence on various properties of the KVS. Some results of the numerical simulation are explained by means of a scale analysis and a qualitative linear stability analysis. Finally, the findings are summarized and useful future investigations are identified.

\section{PROBLEM STATEMENT AND MATHEMATICAL FORMULATION}

In this section, the general features of the flow problem are described and the computational domain, governing equations, boundary conditions and the numerical implementation are specified.

\section{A. Flow configuration}

Since all simulations are carried out in the LVSR, the problem is treated as two-dimensional. The geometry for the flow problem is given in Fig. 1, where the spatial fluid domain and boundary $i$ are denoted as $\Omega$ and $\partial \Omega_{i}$, respectively. The geometry consists of an infinitely long cylinder of diameter $d$ that is symmetrically placed in a plane channel of height $h$. The cylinder is located at a distance of $2 h$ and $8 h$ to the channel inlet and outlet, respectively. Preliminary computations show that this position allows for the use of a fully developed laminar flow profile as an inlet boundary condition. Likewise, we find no influence of the outlet boundary condition on the formation of the KVS. Two electrodes are located at the channel walls in vicinity to the cylinder which allow spanning up an electric field. This induces an electrokinetic flow at the cylinder interface. Note that the position and size of the electrodes are

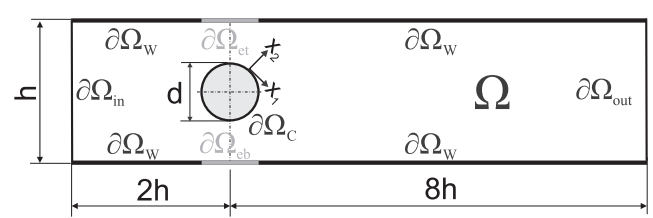

FIG. 1. Schematic of the simulation domain for the confined flow around a cylinder with an exemplary local wall-tangential and -normal coordinate system. Gray lines indicate electrodes whose positions are varied in this study. 
varied to investigate the influence of different electrokinetic manipulations.

\section{B. Mathematical formulation}

We use the following notation: all independent (time and length) and dependent (velocities, pressure, and electric potential) variables have a $\sim$ symbol if they have a physical dimension, otherwise they are dimensionless. The numerical simulation requires the solution of the momentum transport and the electric potential in the domain. Here, the problem arises that the EDL thickness is usually several magnitudes of order smaller than the channel diameter. A first-principle treatment would be very expensive, and we therefore apply the method of matched asymptotic expansions to reduce the numerical costs; the detailed derivation and verification of the model are published elsewhere in Ref. 16.

The governing equations are related to a local tangential and normal coordinate system $\mathbf{x}=\left(x_{1}, x_{2}\right)^{T}$ with the origin at the channel wall. The full model comprises the Poisson equation for the electric potential $\varphi$. The flow $\mathbf{u}=\left(u_{1}, u_{2}\right)^{T}$ and pressure $p$ field of the incompressible Newtonian liquid is described by the time dependent Navier-Stokes equations extended by an electrostatic force term. The variables are nondimensionalized with the electric potential scale $\varphi_{0}$, maximum velocity amplitude $u_{0}$ of the pressure-driven flow, viscous pressure scale $p_{0}=\mu u_{0} / d_{0}$, convective time scale $t_{0}$, and the channel height $h$. The simulation domain can be divided into two subdomains distinguished by whether an electrostatic force acts on the liquid or not; i.e., the electrically neutral bulk liquid and the electrically charged EDL. We find approximate analytical solutions for electric potential, flow, and pressure within the EDL, cf. Ref. 16. These solutions are converted into (transition) boundary conditions for the electrically neutral bulk liquid which has to be solved numerically. In other words, the simulation domain is bounded by the transition of neutral liquid and EDL and not by the channel wall.

In detail, the electric potential is governed by the Laplace equation in conjunction with a Neumann boundary condition that is applied at all boundaries but the electrodes; i.e.,

$$
\begin{aligned}
\Delta \varphi \simeq 0 & \text { in } \Omega, \\
\partial_{x_{2}} \varphi 0 & \text { on } \partial \Omega_{w}, \partial \Omega_{c}, \partial \Omega_{\text {in }}, \partial \Omega_{\text {out }} .
\end{aligned}
$$

At the electrodes, we prescribe Dirichlet conditions:

$$
\varphi=\text { Const } . \quad \text { on } \partial \Omega_{e t}, \partial \Omega_{e b} .
$$

The governing equations of the flow field are the standard incompressible Navier-Stokes and continuity equation

$$
\begin{aligned}
\nabla \cdot \mathbf{u}=0 & \text { in } \Omega, \\
\operatorname{Re}_{c h}\left(\partial_{t} \mathbf{u}+(\mathbf{u} \cdot \nabla) \mathbf{u}\right)-\Delta \mathbf{u}+\nabla p=0 & \text { in } \Omega,
\end{aligned}
$$

where $R e_{c h}=\frac{u_{0} h}{v}$ is the channel Reynolds number and $v$ is the kinematic viscosity of the fluid. In the present case, having an internal flow through the channel and an external flow around the cylinder, both the channel and the cylinder Reynolds number $R e=\varepsilon R e_{c h}$ describe the flow problem adequately. The corresponding boundary conditions at the cylinder and channel
(EDL to bulk liquid) interface are

$$
\begin{aligned}
\mathbf{u} & \simeq-\Pi\left(\partial_{x_{1}} \varphi, 0\right)^{T} & & \text { on } \partial \Omega_{c}, \partial \Omega_{w}, \\
\partial_{x_{2}} p & \simeq 0 & & \text { on } \partial \Omega_{c}, \partial \Omega_{w} .
\end{aligned}
$$

The dimensionless group $\Pi=\frac{l_{D} q_{\zeta} \varphi_{0}}{u_{0} h \mu}$ can be interpreted as the ratio of electric to viscous forces, where $\mu$ denotes the liquid dynamic viscosity, $l_{D}$ denotes the Debye length, and $q_{\zeta}$ denotes the charge density at the shear layer of the EDL. Note that not only parameters like the charge density but also the electric field distribution depends on the properties of the channel material. Assuming common parameters for water paired with a dielectric substrate such as glass having a surface charge that does not arise from (electric) polarization $\left(q_{\zeta}=3.3 \times 10^{-4} \mathrm{C} / \mathrm{m}^{2}, l_{D}=210 \mathrm{~nm}, \mu=0.001 \mathrm{~Pa} \mathrm{~s}\right)$, we compute that $\Pi=0.014$ is a typical ratio of electric to viscous forces in our problem. This value already indicates that we use only small perturbations to influence the flow. The required potential differences between the electrodes are then of the order of magnitude of $10 \mathrm{~V}$ for a channel of $1 \mathrm{~mm}$ height. To summarise, any wall-tangential component of an electric field $\mathbf{E}=-\nabla \varphi$ at the cylinder (EDL to bulk liquid) interface leads to a finite velocity boundary condition. Note that this condition also fulfills the no-slip condition if there is no tangential component of the electric field which is essentially the case for all wetted boundaries but the cylinder. Any wall-normal component of an electric field leads to a pressure change over the thickness of the EDL, cf. Ref. 16. The resulting boundary condition at the interface between EDL and electrically neutral bulk liquid is a constant pressure in the normal direction which still depends on the tangential coordinate; numerically we use the corresponding Neumann condition. Finally, for the inlet boundary we use a fully developed laminar flow profile while a constant pressure along with no viscous stress boundary condition is implemented at the outlet, which is

$$
\begin{array}{ll}
\mathbf{u}-\left(0,4\left(1 / 4-x_{1}^{2}\right)\right)^{T}=0 & \text { on } \partial \Omega_{\text {in }}, \\
p=0,\left(\nabla \mathbf{u}+\nabla(\mathbf{u})^{T}\right) \mathbf{n}=0 & \text { on } \partial \Omega_{\text {out }} .
\end{array}
$$

All simulations start with a fluid at rest as an initial condition. If there is an electrokinetic manipulation, we ramp up the influence of the electric field so that the maximum is achieved at $t=0.1$. For the characterization of the vortex shedding regime, a dimensionless frequency, commonly referred to as the Strouhal number, is used

$$
S t=\frac{\tilde{f} d}{u_{0}}
$$

where $\tilde{f}$ is the dimensional frequency of the alternating shedding vortices. To determine the vortex shedding frequency, it is very common to analyze the lift coefficient

$$
c_{L}=\frac{2\left|\mathbf{F}_{\mathbf{L}}\right|}{\rho u_{0}^{2} d}=\frac{2\left|\oint \tilde{p} \mathbf{n} \partial \Omega_{c}\right|}{\rho u_{0}^{2} d}
$$

or the drag coefficient

$$
c_{D}=\frac{2\left|\mathbf{F}_{\mathbf{D}}\right|}{\rho u_{0}^{2} d}=\frac{2\left|\oint\left(\tilde{p}+\tilde{\tau}_{w}\right) \mathbf{t} \partial \Omega_{c}\right|}{\rho u_{0}^{2} d},
$$


where the pressure distribution and the shear stress $\tilde{\tau}_{w}$ at the cylinder interface are required. However, the EDL thickness in the present numerical simulations is neglected. The pressure in the EDL corresponds to the sum of the pressure at the transition and an additional electrically induced pressure according to $p_{E D L} \simeq p\left(x_{1}, 0\right)+\frac{q_{\zeta}^{2} h}{2 u_{0} \mu \epsilon} \exp \left(\frac{-2 h}{l_{D}} x_{2}\right)$, where $\epsilon$ is the liquid permittivity. ${ }^{16}$ This additional pressure term does neither contribute to the lift nor to the drag force since its integration along a cylinder interface is zero.

\section{Simulation methodology}

Calculations are performed on a Dell Precision Tower 5810 workstation equipped with 1 Quad-core Intel Xeon E5-1620 v4 (3.5 GHz) CPU and $16 \mathrm{~GB}$ of main memory. The governing equations and corresponding boundary conditions are implemented in the Finite Element Method software COMSOL Multiphysics version 4.3b/5.0. To solve the dimensionless model, we adjust the parameters of the implemented dimensional equations by replacing the density with the Reynolds number, set the viscosity to unity, etc. The geometry as given in Fig. 1 is discretized with a regular mesh of quadrilateral elements. In detail, an O-grid is used around the cylinder and all meshes are refined in the vicinity of the boundaries to accurately resolve the enhanced gradients of the flow field. Additionally, the length (aspect ratios) of the elements is varied along the channel with a decreasing length in the vicinity of the cylinder, while the element length increases in the downstream direction. For the fluid flow, a P2 + P1 scheme is applied for the discretization of the conservation equations by Galerkin's method with quadratic and linear functions to interpolate velocity and pressure distributions in the elements, respectively. Quadratic elements are used for the governing equation of the electric potential.

There is a one-way coupling of electric potential and flow field. Therefore, the simulation process is divided into two steps. First, the steady solution of the electric potential is computed which is then used to compute the boundary conditions of the flow field. For both steps, a direct solver is chosen. The stationary step is solved with the MUMPS (multifrontal massively sparse direct solver) algorithm, ${ }^{17}$ while the time dependent step uses the PARDISO (parallel sparse direct linear solver) algorithm. ${ }^{18}$ The implicit second order backward differentiation formula (BDF) ${ }^{19}$ with a upper limit time step of $t=0.01$ is used as a time stepping method.

Convergence of the solution at all time steps is achieved when the Root Mean Square of the residuum, summed up over all equations, remains less than $10^{-4}$. Note that per default, the streamline and crosswind diffusion are enabled in COMSOL for the sake of faster convergence. These stabilization methods result in dampening of instabilities and have to be disabled. Mesh independency studies are performed and we refine the mesh until a $10 \%$ increase of the element number changes the Strouhal number of the vortex shedding by less than $0.2 \%$, which is a good compromise between accuracy and computational costs. Typically, such a mesh has more than 100000 degrees-of-freedom.

\section{RESULTS AND DISCUSSION}

Section III is divided in two subsections. First, we present results for a pure pressure-driven flow around a confined cylinder which serves as validation of the numerical methodology. Next, we discuss results where the confined cylinder flow is subjected to a steady electric field that induces an additional electrokinetic flow. All simulations are performed in the 2D Laminar Vortex Shedding Regime for representative blockage ratios of $\varepsilon=0.1,0.3$, and 0.5 . We also perform a scale analysis to obtain further fundamental insight into the flow phenomena. All Strouhal numbers are based on a Fast Fourier Transformation Analysis (FFT) of the lift coefficient unless otherwise indicated.

\section{A. Pressure-driven flow around a confined cylinder}

Simulations of a pure pressure-driven flow are performed with the aim to infer the critical Reynolds number and the correlation between Reynolds and Strouhal number.

\section{Critical Reynolds number}

The first computations aim to infer the critical Reynolds number $R e_{c r}$, for a given blockage ratio $\varepsilon$, where vortex shedding is observed. Table I summarizes the computed critical Reynolds and the corresponding (critical) Strouhal number $S t_{c r}$ along with the data reported by Chen et al. ${ }^{10}$ and Sahin and Owens. ${ }^{11}$ In their work, the critical Reynolds numbers are inferred by solving the generalized eigenvalue problem of a linear stability analysis.

The critical Reynolds number of an unconfined flow ( $\varepsilon=0$ ) was experimentally found by Williamson ${ }^{20}$ to be $R e_{c r}=48.33$. We observe vortex shedding for $R e_{c r}=51.25$ for the small confinement of $\varepsilon=0.1$. This shift to a higher value can clearly be explained by a damping effect due to the channel walls. In general, we observe that the higher the blockage ratio, the higher the critical Reynolds number. There is a very good agreement of the $R e_{c r}$ values computed in this work and those reported in the literature. Generally, we find values very close to or somewhat less than those of Chen et al. ${ }^{10}$ The difference to the values of Sahin and Owen ${ }^{11}$ is always less than $1 \%$. The comparison of the corresponding critical Strouhal numbers $S t_{c r}$ also shows a very good agreement; though the maximum deviation is at $1.7 \%$ somewhat higher. This is probably related to the FFT where the resolution of the frequency depends on the sample number of the data set and on the implemented algorithm. Note that there is strong indication that we can compute critical Reynolds numbers closer or even identical

TABLE I. Comparison of the computed critical Reynolds and Strouhal numbers with the literature.

\begin{tabular}{lcccc}
\hline \hline Critical number & $\varepsilon$ & Sahin and Owens $^{11}$ & Chen et al. & This work \\
\hline $\operatorname{Re}_{c r}$ & 0.1 & 50.75 & 51.77 & 51.25 \\
$S t_{c r}$ & & 0.121 & 0.112 & 0.119 \\
$R e_{c r}$ & 0.3 & 94.40 & 94.85 & 95.00 \\
$S t_{c r}$ & & 0.209 & 0.209 & 0.210 \\
$R e_{c r}$ & 0.5 & 123.75 & 124.58 & 124.50 \\
$S t_{c r}$ & & 0.339 & 0.338 & 0.341 \\
\hline \hline
\end{tabular}


to the values of Sahin and Owen. ${ }^{11}$ For example, our simulation at $R e=50.75$ and $\varepsilon=0.1$ features a monotonic increase of the lift coefficient amplitude over time. However, even after a 100000 time steps $(t=1000)$ we do not observe the shedding of the vortices. Based on other results, we extrapolate that roughly a time of $t \sim 10000$ is required for the establishment of a fully developed KVS at this Reynolds number. This is not practical for this work since it is not concerned with improving the accuracy of $R e_{c r}$ values reported in the literature. To summarize, our numerical methods are able to reproduce the onset of the KVS with a very good accuracy.

\section{Strouhal number versus Reynolds number}

Further investigations are performed to infer the correlation between Strouhal and Reynolds numbers in the LVSR for different blockage ratios. To get some fundamental insights, a scale analysis of the wake flow is conducted first. For the sake of simplicity, we use dimensional variables. In terms of an unconfined flow, we partly follow the methodology of Dowell and Jaworski ${ }^{21}$ and assume that the fully developed KVS can be expressed with a steady base flow (super indexed ') and a perturbation (super indexed ") of the base flow. Thus, the axial and normal scalar velocity and the pressure are

$$
\tilde{u}_{1}=\tilde{u}_{1}^{\prime}+\tilde{u}_{1}^{\prime \prime}, \quad \tilde{u}_{2}=\tilde{u}_{2}^{\prime}, \quad \tilde{p}=\tilde{p}^{\prime}+\tilde{p}^{\prime \prime} .
$$

A homogeneous scaling is introduced, where all (dimensional) spatial and time derivatives are assumed to be of the following order:

$$
\frac{\partial}{\partial \tilde{x}_{1}}, \frac{\partial}{\partial \tilde{x}_{2}} \sim \frac{1}{d}, \quad \frac{\partial}{\partial \tilde{t}} \sim \tilde{f} .
$$

Relations (13) and (14) are introduced in the (dimensional) equation of the axial momentum transport, and it is further assumed that the unconfined base flow velocity $\tilde{u}_{1}{ }^{\prime}$ is similar to the convective velocity scale $u_{0}$. We first compare the viscous and the convective terms. It turns out that for the Reynolds number range of interest, the viscous term can be neglected. Comparing the transient term with a convective term results in

$$
\frac{\tilde{f} d}{u_{0}} \equiv S t \sim 1,
$$

if $O\left(\tilde{u}_{1}^{\prime \prime}\right) \leq O\left(u_{0}\right)$ which is always the case as we can see in the numerical results.

In terms of a confined flow, we realize that the maximum velocity in the vicinity of the cylinder can be quite different compared to the convective velocity scale (inlet flow amplitude). Hence, we introduce the gap velocity $u_{\varepsilon} \sim u_{0} /(1-\varepsilon)$ and repeat the scale analysis in a similar manner as for the unconfined flow but using the gap velocity. Then, the Strouhal number of the confined cylinder flow is similar to

$$
S t \sim \frac{1}{1-\varepsilon} .
$$

We perform a set of simulations to infer the correlation between Strouhal and Reynolds numbers in the LVSR for different blockage ratios. Figure 2 summarizes the simulation results and compares them to the experiments of Williamson ${ }^{20}$ for $\varepsilon=0$ as well as to $R e_{c r}$ computed by Sahin and Owens ${ }^{11}$ (vertical dashed lines). For a constant Reynolds number and an increasing blockage ratio, the Strouhal number noticeably

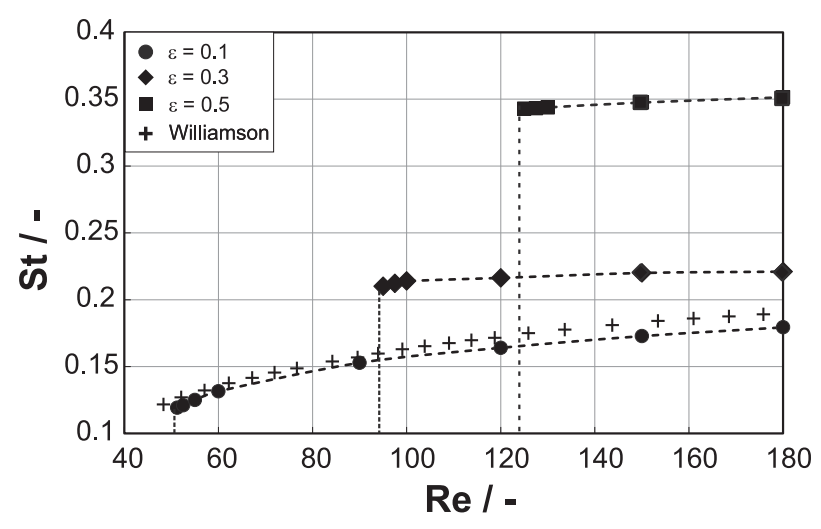

FIG. 2. Strouhal number versus the Reynolds number. Comparison of the present study (solid markers) with experiments of Williamson ${ }^{20}$ (+marker) for an unconfined pressure-driven flow. Connection between data points are guides for the eyes. Vertical lines indicate critical Reynolds numbers as computed by Sahin and Owens. ${ }^{11}$

increases. Given a constant blockage ratio, there is only a relatively small increase of the Strouhal number when the Reynolds number increases. This small increase is more pronounced for the lowest blockage ratio of $\varepsilon=0.1$, while for $\varepsilon=0.3$ and 0.5 the Strouhal number remains almost constant. The comparison with the experimental data for $\varepsilon=0$ clarifies that even the small confinement of $\varepsilon=0.1$ results in a higher critical Reynolds number. The difference between the Strouhal numbers is relatively small with the unconfined flow being shifted to slightly higher values; the maximum difference is only around 3\%. Our computations also show a good agreement with some of the results of Griffith et al. ${ }^{14}$ (data not shown). These authors also performed 2D computations of the flow past a confined cylinder and infer the Strouhal number of the vortex detachment as a function of the Reynolds number. The deviations between the Strouhal numbers, for a given Reynolds number, in that work and ours are around or less than $2 \%$. However, in that work considerably lower critical Reynolds numbers for the blockage ratios of $\varepsilon=0.3$ and 0.5 are reported compared to Refs. 10 and 11 and the present work.

If we quantitatively compare the results of scale analysis and computations, there is only moderate agreement. The computed Strouhal numbers are rather $O(S t)=0.1$, while the scale analysis predicts $O(S t)=1$. Nevertheless, in terms of qualitative agreement the scale analysis reflects the simulation results very well: On the one hand, the Strouhal number is mainly independent of the Reynolds number. That is, the order of magnitude of the Strouhal number is constant within a Reynolds number range having a constant order of magnitude. On the other hand, a plot of the computed Strouhal number versus the blockage ratio at a given Reynolds number (not shown) recovers a proportionality which is similar to $1 /(1-\varepsilon)$.

To summarize, we find a very good agreement between the (critical) Reynolds and Strouhal numbers computed in this work and those reported in Refs. 10, 11, and 14 confirming the choice of the numerical schemes and parameters. Simulation results show that the confinement increases the dimensionless vortex shedding frequency, while a scale analysis clarifies that this is related to increased gap velocity around the cylinder. 


\section{B. Electrokinetic manipulation with a DC electric field}

Next, we investigate the influence of a DC electric field acting on the cylinder. It is hypothesized that the induced electrokinetic manipulation in the vicinity of the cylinder influences the properties of the vortex shedding. First, we introduce the different investigated electrode configurations and discuss their influence on the flow topology around the cylinder. Then, we analyze the lift coefficient $c_{L}$ and the drag coefficient $c_{D}$ since they are integrals of the local shear and pressure forces that act in parallel and normal direction to the cylinder surface. Finally, the influence of the electrokinetic manipulations on the vorticity in the cylinder wake is investigated.

\section{Electrical configurations and resulting manipulations}

Figure 3 comprises the three general electrode configurations that we investigate where the upper row illustrates the different position of the electrodes (black bars) relative to the cylinder. Note that we also vary the electrode polarity so that the electric field changes its direction. The lower row shows the electric field distribution resulting from numerical simulations. For the sake of a better description, we introduce an angular coordinate $\alpha$ starting from the front of the cylinder in the clockwise direction. Hence, the indicated positions I, II, III, and IV correspond to an angle of $0^{\circ}, 90^{\circ}, 180^{\circ}$, and $270^{\circ}$, respectively. In detail, Fig. 3(a) gives the configuration S1 where the electrode centers are aligned with the vertical cylinder centerline. The electrode polarity is chosen such that the induced electric field points mainly upwards; this configuration is accordingly labeled S1U in this manuscript. Here, the highest electric field strengths can be found at cylinder position I and III, while the lowest field strengths are at II and IV. Recall that the induced electrokinetic flow at the cylinder surface is directly proportional to the electric field, cf. Eq. (6). For a pure pressure driven flow, locations I and III correspond to the forward and rear stagnation point, respectively. In the present case, we find the strongest electrokinetic manipulation there while there is no electrokinetic flow at II and IV. In other words, the electrokinetic stagnation points are shifted for $90^{\circ}$ compared to the pressure-driven stagnation points. Eventually, it is easier to interpret the influence of the electric field distributions on the cylinder when they are expressed by an equivalent (integral) electrostatic force $\mathbf{F}_{\mathbf{e}}=q \phi \mathbf{E} \partial \Omega_{c}$. In the present case where the field is symmetric with respect to the vertical cylinder centerline, the resulting equivalent electrostatic force solely acts on the cylinder in the vertical direction.

Figure 3(b) shows a sketch of configuration S2 where the electrodes are displaced along the channel wall so that the inner electrode edges align with the vertical cylinder centerline. Two different polarities are investigated for this electrode configuration. Configuration S2D is the polarity where the resulting electric field goes from the upper electrode down to the lower electrode. The polarity with the field in the opposite (up) direction is accordingly labeled S2U. The electric field distribution for case S2D indicates that the electrokinetic stagnation points are shifted around $45^{\circ}$ compared to the pressure-driven stagnation points. Case $\mathrm{S} 2 \mathrm{U}$ is equal in terms of distribution but the field is in the reverse direction. In contrast to the previous case $\mathrm{S} 1$, the equivalent electrostatic force acting on the cylinder has components in vertical and horizontal directions. The horizontal component points in the down- and upstream direction for electrokinetic manipulation S2D and S2U, respectively.

Figure 3(c) sketches the electrode configuration S3 where electrodes are virtually positioned at the channel in- and outlet. That is, we just prescribe respective potentials at $\partial \Omega_{i n}$, $\partial \Omega_{\text {out }}$, but there is no influence on the flow field. Such configurations can be realized by immersion of the electrodes in the reservoirs that are connected to the channel in order to supply and collect the liquid. The two different electrode polarities that are investigated result in electric fields that point mainly in the down- (S3D) or upstream (S3U) direction. Then, the electrokinetic stagnation points for both configurations coincide with the pressure-driven stagnation points. It is obvious that the electric field distribution in both cases is symmetric with respect to the horizontal cylinder centerline. Thus, the equivalent electrostatic force has no vertical component and acts solely in the downstream (S3D) or upstream (S3U) direction.

Additionally, we observe that all configurations induce electrokinetic flows close to the separation points which are roughly located at $130^{\circ}$ and $230^{\circ}$ for the conventional LVSR. However, some electrode configurations equally influence

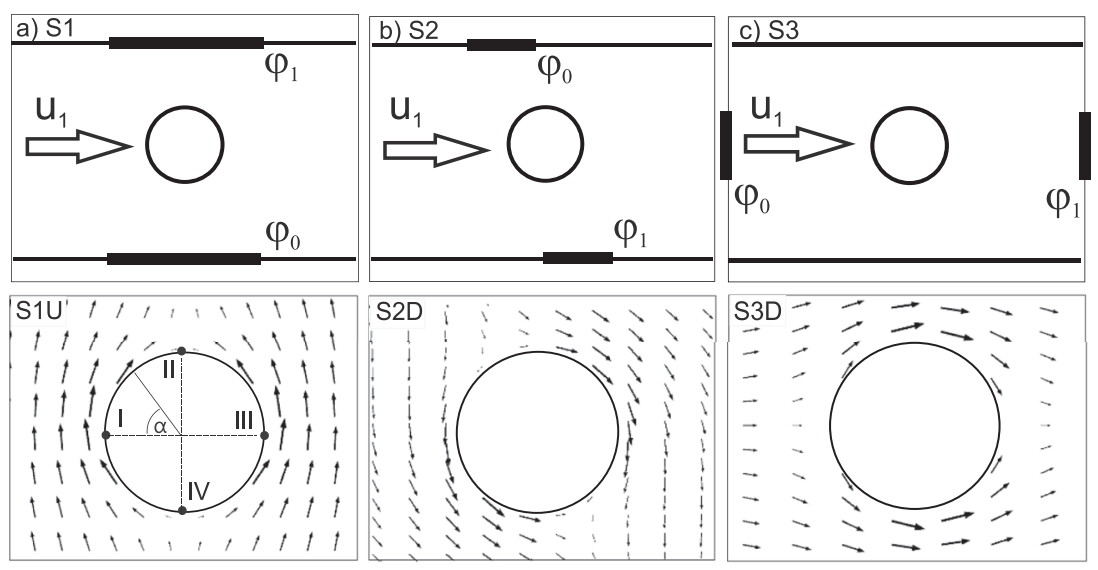

FIG. 3. Sketch of electrode positions (upper row) and resulting electric field distributions (lower row): (a) Configuration $\mathrm{S} 1$ where electrode centers are aligned with the vertical cylinder centerline; (b) Configuration S2 where electrode centers are out of alignment; (c) Configuration $\mathrm{S} 3$ where electrodes are (virtually) located at the channel inlet and outlet, respectively. 
both separation points, while others manipulate mainly one. For example, S3D induces similar electrokinetic flows at the upper and at the lower separation point. By contrast, case S2D induces a considerable electrokinetic velocity at the upper separation point, but there is practically no electrokinetic flow at the lower one.

Figure 4 shows the shear stresses along the cylinder perimeter for a flow with $R e=60, \Pi=0.014$, and $\varepsilon=0.1$ without and with different electrokinetic manipulations. We choose early simulation times around $\tau \approx 5$, where the amplitude of the developing lift coefficient is zero and the vortices have not yet detached from the cylinder. In other words, the flow is still in a "temporary" Laminar Steady Regime while transitioning to the LVSR. This situation allows for an insight into how the electrokinetic manipulation influences the flow topology around the cylinder and the development of the VKS. In detail, part (a) shows the wall shear stress at the cylinder surface for the pure pressure driven flow. It is right away obvious that the shear stress distribution is symmetric with respect to the horizontal centerline of the channel. The wall shear stress is zero at the forward and rear stagnation point and rises to a maximum at around $50^{\circ}$ and $310^{\circ}$. There are two other locations at $120^{\circ}$ and $240^{\circ}$ with zero shear stress indicating the locations (saddle points) where the counter-rotating recirculation flow in the rear meets the downstream flow. Figure 4(b) reveals the influence of the different electrokinetic manipulations on the wall shear stress. For the sake of a better illustration, we solely focus on the rear of the cylinder. This is also the region with the highest influence of the manipulation since there are the lowest (pressure-driven) flow velocities. Inspection of the figure clarifies that all cases with a vertical equivalent electric field component (S1U, S2D, and S2U) have a nonsymmetric wall shear stress distribution. It is noteworthy that S1U, having the equivalent electrostatic force only in the vertical direction, features a rear stagnation point at $183^{\circ}$ with a zero wall shear stress, while the others have finite minimum values. All electrokinetic manipulations with a sole equivalent electrostatic force in the horizontal direction (S3D and S3U) maintain the symmetry but do not have a location with zero wall shear stress.

Next, we inspect the flow topologies in the vicinity of the cylinder for the same conditions as for Fig. 4. For this purpose, we compute instantaneous streamlines where we always use the same (seeding) procedure so that differences in topology are only related to the different electrokinetic manipulations. Figure 5(a) shows the flow topology of the pure pressure-driven flow (NF). At these conditions, the flow field, like the wall shear stress, is symmetric with respect to the horizontal centerline of the channel. We clearly identify the forward stagnation point I where the flow symmetrically branches. The streamlines follow the cylinder geometry until they meet at the separation points, located at around $\alpha=115^{\circ}$ and $205^{\circ}$, with those of the counter-rotating recirculation zone and separate from the cylinder. In the cylinder wake, the fluid is transported along the horizontal centerline toward the rear stagnation point III. From there, the flow branches and the streamlines follow the cylinder geometry toward the separation points.

Figure 5(b) shows the flow topology for the same pressuredriven flow with the additional electrokinetic manipulation S1U; the corresponding electrokinetic flow can be qualitatively inferred from Fig. 3(a). There are distinct differences compared to the pure pressure-driven flow. As can be seen at the front and rear stagnation points, the electrokinetic flow breaks the symmetry of the streamlines which is present for a pure pressure-driven flow prior to vortex shedding. In other words, streamlines originating from the upper channel half cross the horizontal (symmetry-) centerline and move into the lower channel half. This asymmetry is more pronounced, as discussed above for the wall shear stress, and the streamlines are more distorted at the rear stagnation point. We also observe that this electrokinetic manipulation influences both separation points by inducing electrokinetic flow in the direction of $-\alpha$. Inspection of the flow topologies of cases S2D and S2U, given in Figs. 5(c) and 5(d), indicates that these manipulations break the (initial) flow symmetry as well. Close to the stagnation points, the fluid is pulled across the channel centerline in the upper or lower direction depending on the direction of the electric field. We note that this displaced electrode arrangement, for either the electric field direction, influences mainly the flow topology at the upper separation point since the lower one is close to the electrokinetic stagnation point.

By contrast, both electrokinetic manipulations S3D and S3U maintain the early-on flow symmetry as can be seen in Figs. 5(e) and 5(f). Nevertheless, a detailed inspection reveals
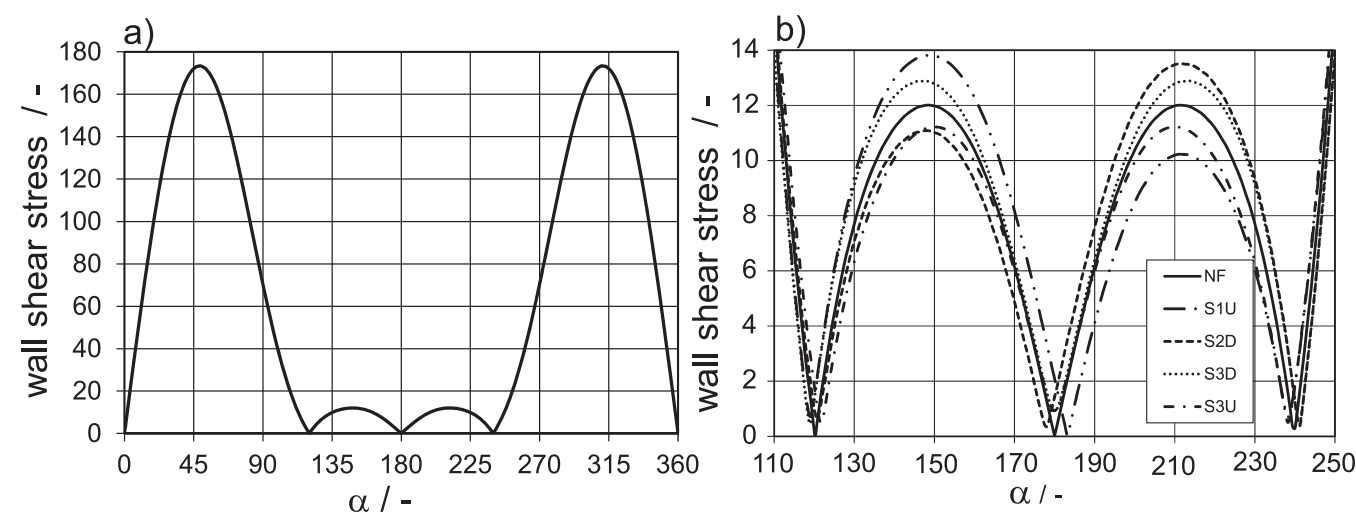

FIG. 4. Wall shear stress at the cylinder before vortex shedding at $R e=60, \Pi=0.014$, and $\varepsilon=0.1$ : (a) Distribution along the entire cylinder perimeter for case $\mathrm{NF}$ (no electric field) and (b) distribution in the rear of the cylinder for cases NF, S1U (aligned electrodes, field up), S2D (displaced electrodes, field down), S2U (displaced electrodes, field up), S3D (field downstream), and S3U (field upstream). 

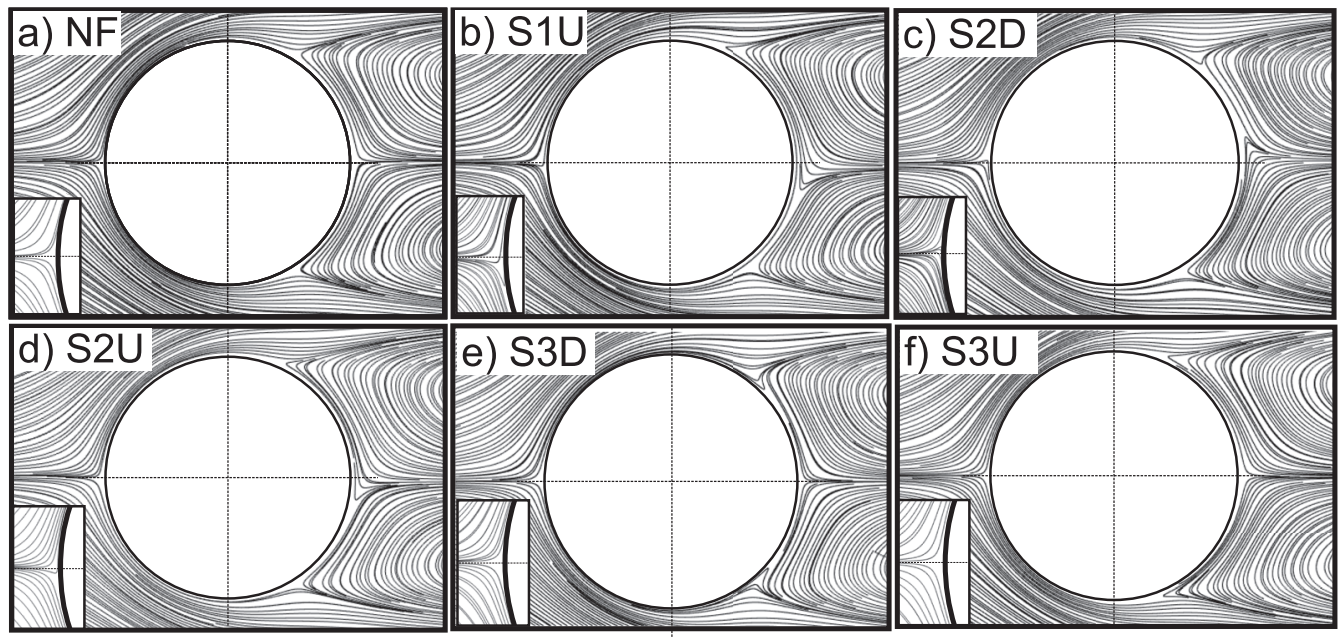

FIG. 5. Instantaneous streamlines close to the cylinder surface before vortex shedding at $R e=60, \Pi=0.014$, and $\varepsilon=0.1$ for different electrokinetic manipulations: (a) NF (no electric field); (b) S1U (aligned electrodes, field up); (c) S2D (displaced electrodes, field down); (d) S2U (displaced electrodes, field up); (e) S3D (field downstream); (f) S3U (field upstream). The insets are magnifications of the forward stagnation point I.

that there is a difference in terms of the flow topology close to the separation points. In contrast to the displaced electrode configuration, the upper and lower separation point is equally influenced. For manipulation S3D, the electric field in the downstream direction shifts both separation points toward the rear stagnation point. By contrast, the upstream-directed electric field in case S3U results in a shift toward the front stagnation point. Eventually, the comparison of all flow topologies confirms and expands the findings of the wall shear stress. Only electric field distributions which induce an equivalent electrostatic force with a component in the vertical direction break the initial flow symmetry. Nevertheless, we can still expect deviations in the KVS characteristics between configurations with maintained symmetry since the separation and rear stagnation points (cf. discussion wall shear stress) are influenced.

\section{Lift coefficient}

The vertical force component resulting from the pressure distribution around the cylinder, commonly referred to as the lift coefficient, is extracted from the simulation results using Eq. (11).

Figure 6 shows the temporal evolutions of the lift coefficient for a confined flow of $R e=60$ and $\varepsilon=0.1$ and for different electrokinetic manipulations. In detail, Fig. 6(a) shows the lift coefficient for the case NF without electrokinetic manipulation. The lift coefficient amplitude slowly increases over time until a steady state oscillation is achieved. The oscillations for the different electrokinetic manipulations depicted in parts (b)-(f) have quite diverse appearances compared to the pure pressure-driven flow. Directly obvious is that all manipulations resulting in a vertical equivalent electrostatic force component shift the oscillation to higher or lower values depending on the electrode polarity. This is understood since the lift coefficient represents forces in the perpendicular direction to the main flow.

To obtain a better comparability, we compute integral characteristic parameters of the lift coefficient oscillation. It turns out that the evolution of the amplitude can be fitted to the logistic function $\alpha_{0}+\alpha_{1} /\left(1+\alpha_{2} \exp \left(-\alpha_{3} t\right)\right)$ with very high accuracy. Here, $\alpha_{0}$ and $\alpha_{1}$ correspond to the average and the amplitude of the steady-state oscillation, respectively. We use the point of intersection of the tangent of the inflection point and the tangent of the steady-state amplitude to infer the characteristic (settling) time $t_{s}$ of the oscillation. Starting from a fluid at rest, this is the time that it takes until the KVS is fully developed as indicated by a constant lift coefficient amplitude and frequency.

The regression and characteristic parameters for all cases depicted in Fig. 6 are summarized in Table II. The electrokinetic manipulation has a strong impact on the settling time of the oscillation. For example, while the settling time of the pure pressure-driven flow is $t_{s}=26.4$, the electrokinetic manipulation S1U reduces it to 13.9. A more detailed inspection reveals that only manipulations featuring a vertical equivalent electrostatic force component reduce the settling time. That is, the cases S1U, S2U, and S2D where we also observe the symmetry breaking of the initial flow around the cylinder as discussed in Sec. III B 1. By contrast, when the equivalent electrostatic force is solely directed in the horizontal direction (symmetry maintained), the settling time is similar (S3U) or considerably higher (S3D).

The inspection of the amplitude of the steady state oscillation reveals a more complex behavior. The cases with the pure pressure driven flow NF and the manipulation S1U, with the equivalent electrostatic force only in the vertical direction, have practically the same amplitude. Comparison of S2D and S2U indicates a respective amplitude shift of -0.007 and 0.008 compared to the case NF. Similar behavior is found for case S3D and S3U having a shift of -0.02 and 0.02 , respectively. The magnitude and the sign of the shifts clarify that the lift coefficient amplitude is solely triggered by the horizontal equivalent electrostatic force component. Finally, comparison of the Strouhal number of the steady state lift coefficient oscillations shows that there is no influence of the electrokinetic manipulation. The very minor differences are probably related to the limited accuracy of the FFT. 
a) NF
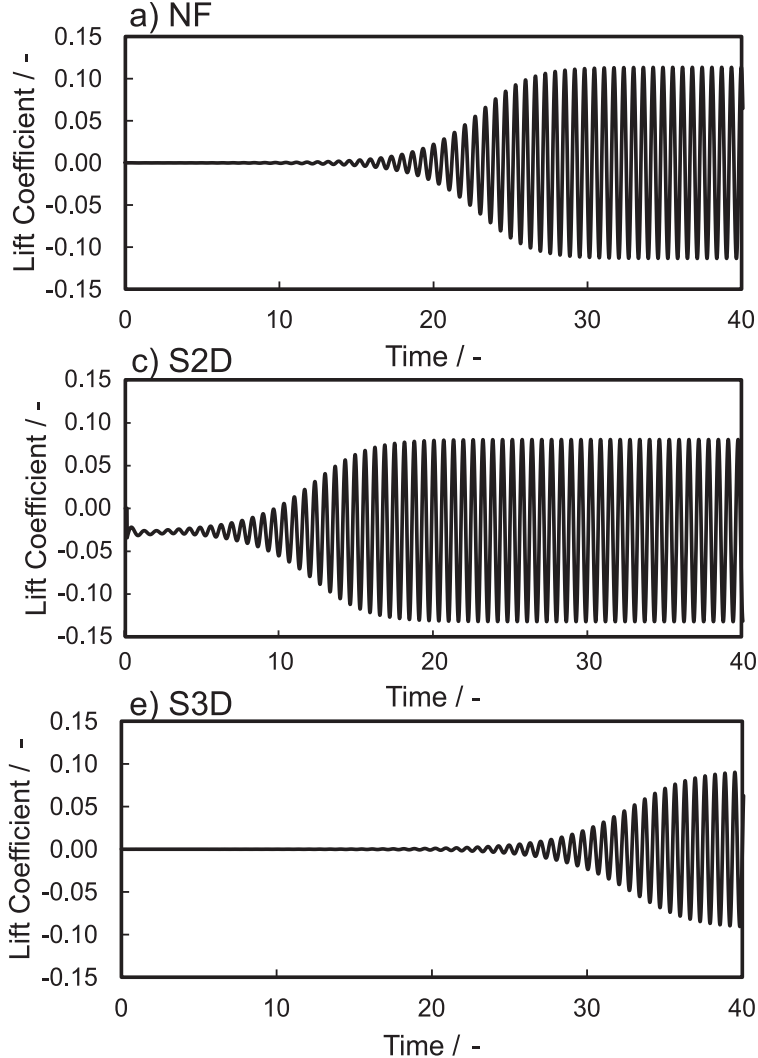

b) S1U
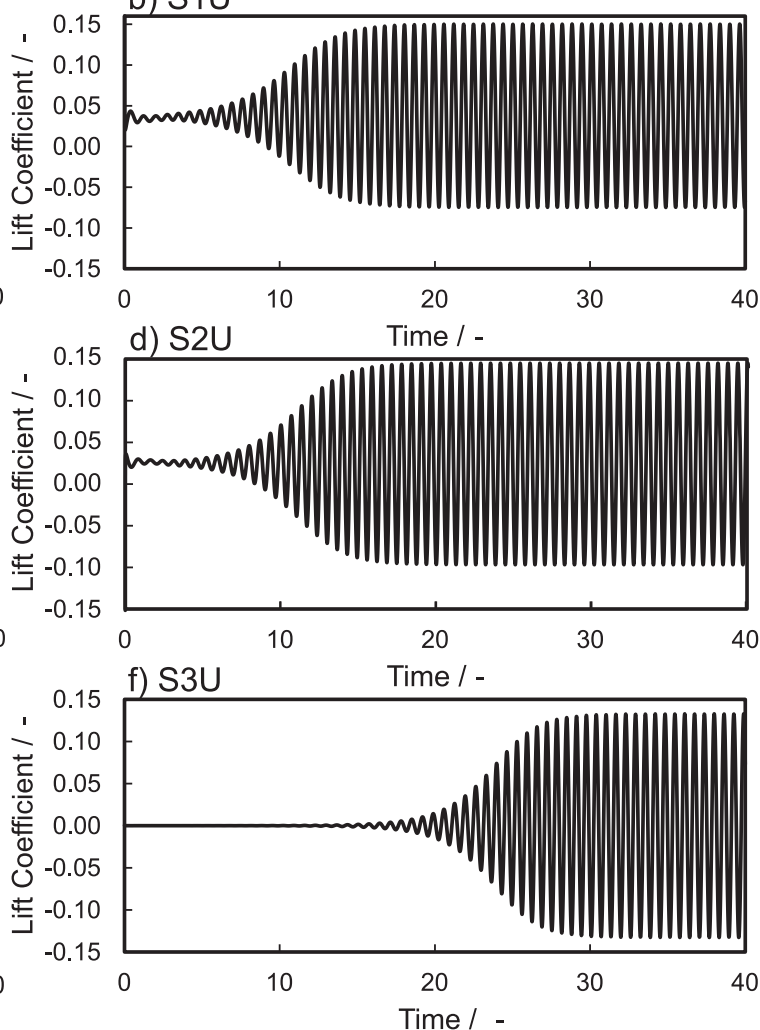

FIG. 6. Lift coefficient versus time for a flow with $\varepsilon=0.1, \Pi=0.014$, and $R e=60$ for different electrokinetic manipulations: (a) NF (no electric field); (b) S1U (aligned electrodes, field up); (c) S2D (displaced electrodes, field down); (d) S2U (displaced electrodes, field up); (e) S3D (field downstream); (e) S3U (field upstream).

To summarize, electrokinetic manipulations with a vertical equivalent electrostatic force component, that break the initial symmetry of the flow around the cylinder, considerably reduce the settling time compared to the pure pressuredriven flow and also shift the oscillation on the $y$-axis. By contrast, the magnitude of the steady state oscillation amplitude is only influenced by the horizontal equivalent electrostatic force component. There is no correlation between the shedding frequency and the application of the DC electric field.

These results demonstrate that small perturbations profoundly influence the transient and the steady state characteristics of the KVS. A natural question arises-when is the perturbation too small to have an impact? Hence, we perform further simulations for a flow with $R e=60, \varepsilon=0.1$, and the electrokinetic manipulation S2U where we vary the

TABLE II. Regression parameters, settling time, (steady-state) amplitude, and Strouhal number of the lift coefficient oscillation for different electrokinetic manipulations at $R e=60, \Pi=0.014$, and $\varepsilon=0.1$.

\begin{tabular}{lcccccc}
\hline \hline Electrode configuration & NF & S1U & S2D & S2U & S3D & S3U \\
\hline$\alpha_{0}$ & 0.000 & 0.037 & -0.027 & 0.028 & 0.000 & 0.000 \\
$\alpha_{2} / 10^{5}$ & 2.537 & 0.003 & 0.006 & 0.008 & 20.306 & 28.163 \\
$\alpha_{3}$ & 0.547 & 0.541 & 0.530 & 0.615 & 0.447 & 0.630 \\
$t_{s}$ & 26.4 & 13.9 & 15.7 & 14.2 & 36.9 & 26.7 \\
$\hat{c}_{L}\left(\alpha_{1}\right)$ & 0.113 & 0.112 & 0.106 & 0.121 & 0.093 & 0.133 \\
$S t$ & 0.132 & 0.131 & 0.132 & 0.132 & 0.132 & 0.131 \\
\hline \hline
\end{tabular}

value of the $\Pi$ group. The results are given in Fig. 7 in the form of relative settling times, i.e., with respect to the settling time of the corresponding pure pressure-driven flow with $\Pi=0$. As expected, these simulations show that the impact on the settling time decreases when the $\Pi$ group - the ratio of electric to viscous forces-decreases. However, it can be seen that there is still a noticeable influence for small and even very small values of the $\Pi$ group. A manipulation with $\Pi \approx 0.001$ considerably reduces the relative settling time to around 0.8 . Even for a value of $\Pi \approx 5 \times 10^{-5}$, a noticeable reduction to 0.95 is computed.

We further investigate the influence of the Reynolds number, blockage ratio, and electrokinetic manipulation on the settling time but restrict the simulations to the cases NF, S1U, S2D, and S2U. Figure 8(a) illustrates the settling times of a

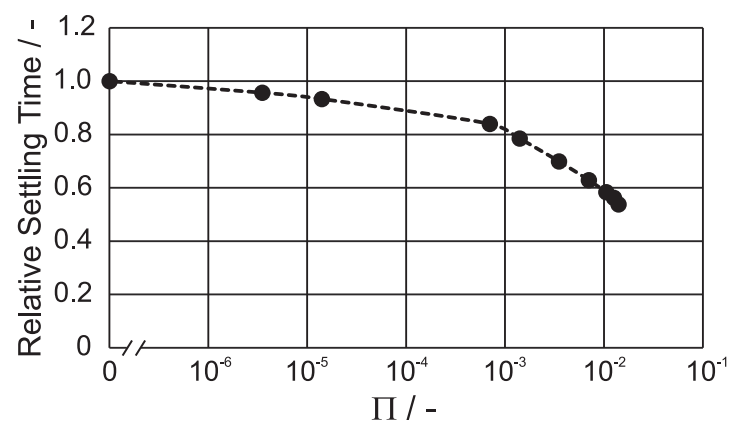

FIG. 7. Relative settling time versus $\Pi$ group for a flow with $R e=60$, $\varepsilon=0.1$, and electrokinetic manipulation $\mathrm{S} 2 \mathrm{U}$. The line is guide to the eyes. 

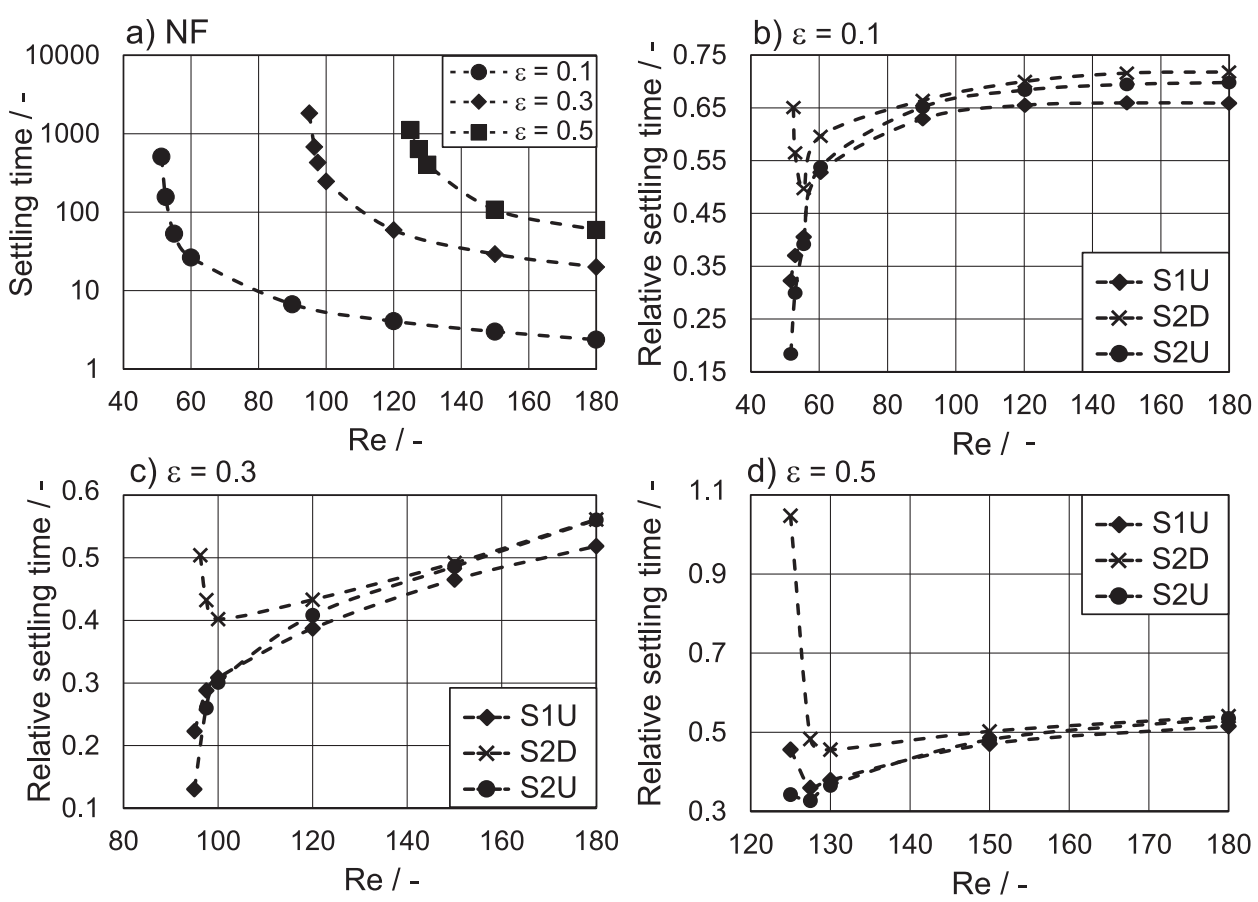

FIG. 8. Settling time of the lift coefficient versus Reynolds number: (a) absolute settling time for pure pressuredriven flow (NF); relative (to NF) settling time for different electrokinetic manipulations with $\Pi=0.014$ and for blockage ratios $\varepsilon$ of (b) 0.1 , (c) 0.3 , and (d) 0.5. Lines are guides to the eyes. pressure-driven flow (NF) with different Reynolds numbers and blockage ratios. For a given blockage ratio, we observe that the highest settling times are observed close to the critical Reynolds number. When the Reynolds number increases, the settling time significantly decreases. The variation spans three orders of magnitude. We further observe that for a constant Reynolds number, the settling time increases with the increase of the blockage ratio.

Figure 8(b) depicts the influence of the different electrokinetic manipulations on the settling time for flows with the blockage ratio $\varepsilon=0.1$. To facilitate the interpretation, we give relative settling times by scaling the results with the respective settling time of the pure pressure-driven flow. We observe that all electrokinetic manipulations considerably reduce the settling time over the entire Reynolds number regime. Here, configuration S1U has the highest impact resulting in the lowest relative settling times compared to the other manipulations. For cases S1U and S2D, we find a continuous increase of the relative settling time when the Reynolds number increases. For case $\mathrm{S} 1 \mathrm{D}$, however, a local minimum around $R e \approx 55$ is observed indicating a damping by this electrokinetic manipulation at very low Reynolds numbers of the LVSR. Indeed, here we do not observe an onset of the KVS at Reynolds numbers close to the critical one. For example, when we analyze the transient lift coefficient for manipulation S1D and $R e=51.25$ (not shown), we observe that the amplitude approaches a maximum relatively early and then asymptotically decreases over time. No vortex detachment is observed, even after very long simulation times, and it seems that the critical Reynolds number is increased by this manipulation. For now, we abstain from further investigations of this phenomenon since the computational cost to determine the critical Reynolds numbers is very high. Nevertheless, this is in stark contrast to the other cases S1U and S2U where the relative settling time is consistently decreasing with decreasing Reynolds number. For example, at $R e=51.25$, the electrokinetic manipulation $\mathrm{S} 2 \mathrm{U}$ reduces the settling time to $17 \%$ of the respective pressure-driven flow. Moreover, further computations for S2U reveal an onset of the KVS at a value of $R e=50$ which is below the critical Reynolds number computed by Sahin and Owens. ${ }^{11}$

Figure 8(c) shows the relative settling times for the blockage ratio $\varepsilon=0.3$. Any electrokinetic manipulation reduces the relative settling time over the entire Reynolds number range. There is again a minimum for case S2D, and the relative settling time increases again when the Reynolds number approaches the critical values. Generally, we observe similar behavior as for the less confined flow but the influence at higher Reynolds numbers is more pronounced and results in somewhat lower relative settling times.

Finally, Fig. 8(d) shows the relative settling times for flows with $\varepsilon=0.5$. In contrast to the other confinements, all electrokinetic manipulations reveal a minimum settling time close to the critical Reynolds number. Manipulation S2D even features a relative settling time larger than one. In other words, it takes longer than the corresponding flow without electrokinetic manipulation. This shows that the same small perturbation can, depending on the Reynolds number, accelerate or damp the temporal evolution of the VKS. Generally, we assume that for all cases with a minimum, an explicit damping with values larger than one could be computed. However, these computations take a very long time and are based on guesses of the respective Reynolds numbers.

We also investigate the influence of the electrokinetic manipulation and the blockage ratio on the amplitude of the steady state lift coefficient oscillation. The results are compiled in Fig. 9 where part (a) shows the influence of the Reynolds number and blockage ratio on the pure pressure-driven flow (NF). For a constant blockage ratio, the amplitude increases as 
a) $\mathrm{NF}$
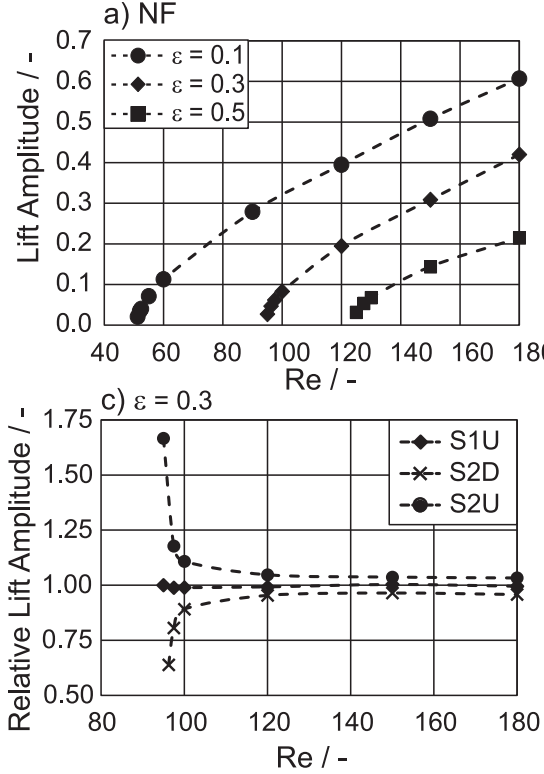

b) $\varepsilon=0.1$

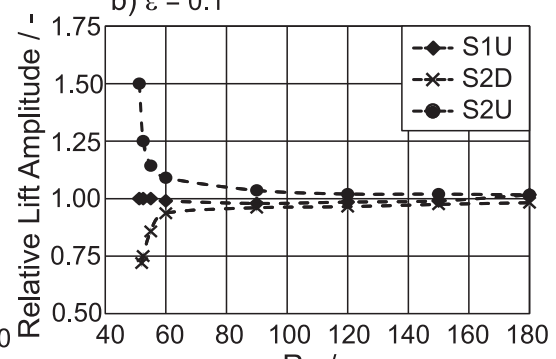

$\mathrm{Re} /$ -

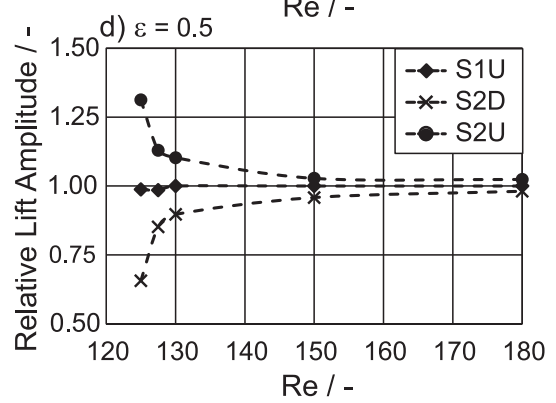

FIG. 9. Steady state amplitude of the lift coefficient versus Reynolds number for different electrokinetic manipulations and blockage ratios: (a) absolute amplitude for pure pressure-driven flow (NF); relative (to NF) amplitude for different electrokinetic manipulations with $\Pi=0.014$ and blockage ratios $\varepsilon$ of (b) 0.1 , (c) 0.3 , and (d) 0.5 . the Reynolds number increases. For a constant Reynolds number, there is a decrease of the amplitude when the blockage ratio increases which can be attributed to the damping effect of the confinement. Part (b) compares the influence of the different electrokinetic manipulations at $\varepsilon=0.1$ in the form of relative lift amplitudes. That is, the amplitudes are scaled with those of the pure pressure-driven flow at the same Reynolds number. While for higher Reynolds numbers, the influence of the different electrokinetic manipulations is getting very small, there can be distinct differences close to the critical Reynolds number.

For case S1U, having a sole vertical equivalent electrostatic force, the relative lift amplitude is always around one and therefore equal to that of the pure pressure-driven flow. For case S2U, having a force component in the upstream direction, the relative lift amplitude is increasing significantly for low Reynolds numbers of the LVSR. The maximum value is around 1.5 at $R e=51.25$. By contrast, the inversion of the electric field (case S2D) results in a damping effect and the amplitude decreases when we approach the critical Reynolds number.

The same behavior is found for the other blockage ratios as can be seen in parts (c) and (d) of Fig. 9. Plotting of the absolute amplitudes over the Reynolds numbers at a constant blockage ratio reveals that all curves have the same form. We do not include these plots since the absolute difference between the curves is rather small and the following is better illustrated with the drag coefficient as shown below. A simple coordinate transformation $R e_{1}=\frac{R e_{0}}{1 \pm \Pi \cos (\varepsilon)}$ is found, where $R e_{0}$ is the Reynolds number of the pure pressure-driven flow and $R e_{1}$ of the electrokinetic manipulation, which results in an overlap of the FED (S2D) and FEU (S2U) data with the curve of the pure pressure-driven flow. The appearance of the cosine in the scaling law confirms that this shift is related to the horizontal component of the equivalent electrostatic force. A component in the downstream and upstream direction decreases and increases the Reynolds number, respectively. In other words, the electrokinetic manipulation S2D results in a lift coefficient that corresponds to that of a pure pressure-driven flow with a lower Reynolds number which explains the damping effects that we observe. If the shift is large enough to place the manipulated flow in the laminar steady regime, we find a suppression of the vortex detachment even though the flow is nominally above the critical Reynolds number (of a corresponding pure pressure-driven flow).

\section{Linear stability analysis}

The purpose of this section is to qualitatively relate the settling times displayed in Table II to the spatial symmetry of the electrode configurations shown in Fig. 3. To this end, we consider a global streamwise-spanwise cartesian coordinate system $\left(x_{1}, x_{2}\right)$ centered at the cylinder axis. The flow develops from an initial configuration $\mathbf{u}_{0}$ that is symmetric with respect to the mirror reflection $x_{2} \rightarrow-x_{2}$ to a von Kármán vortex street that breaks the mirror-reflection symmetry. Here, we consider the initial stages of the flow development in which the flow field has the form $\mathbf{u}=\mathbf{u}_{0}+\delta \mathbf{u}$, where $\delta \mathbf{u}$ is a small perturbation of the initial flow configuration. Keeping terms up to linear order in $\delta \mathbf{u}$ in the Navier-Stokes equation, we obtain

$$
\begin{aligned}
& R e_{c h}\left[\partial_{t}\left(\mathbf{u}_{0}+\delta \mathbf{u}\right)+\left(\mathbf{u}_{0} \cdot \nabla\right) \mathbf{u}_{0}+\left(\mathbf{u}_{0} \cdot \nabla\right) \delta \mathbf{u}+(\delta \mathbf{u} \cdot \nabla) \mathbf{u}_{0}\right] \\
& -\Delta\left(\mathbf{u}_{0}+\delta \mathbf{u}\right)+\nabla\left(p_{0}+\delta p\right)=\mathbf{f}_{e}
\end{aligned}
$$

In this expression, $p_{0}$ and $\delta p$ are the pressure fields corresponding to the initial configuration and the perturbation, respectively. The volumetric force $\mathbf{f}_{\mathbf{e}}$ is due to the electric field acting on the near-wall regions of the fluid carrying a net charge. While in the rest of this manuscript, $\mathbf{f}_{\mathbf{e}}$ is translated into a wall boundary condition, we keep its original form in this section. Considering that $\mathbf{u}_{0}$ is a-yet unstable-solution of the Navier-Stokes equation, we obtain from Eq. (17),

$\operatorname{Re}_{c h}\left[\partial_{t} \delta \mathbf{u}+\left(\mathbf{u}_{0} \cdot \nabla\right) \delta \mathbf{u}+(\delta \mathbf{u} \cdot \nabla) \mathbf{u}_{0}\right]-\Delta \delta \mathbf{u}+\nabla \delta p=\mathbf{f}_{\mathbf{e}}$

To determine the perturbation velocity, this equation has to be solved together with the continuity equation $\nabla \cdot \delta \mathbf{u}$ for a given initial flow field $\mathbf{u}_{0}$. For what follows, it is convenient 
to introduce the parity operator $\mathbf{P}_{x_{2}}$ that causes the mirror reflection with respect to the plane $x_{2}=0$. Under the action of $\mathbf{P}_{x_{2}}$, the spatial coordinates are transformed according to

$$
\mathbf{P}_{x_{2}}\left[\left(x_{1}, x_{2}\right)\right]=\left(x_{1},-x_{2}\right),
$$

while the velocity field transforms according to

$$
\mathbf{P}_{x_{2}}\left[\left(u_{1}\left(x_{1}, x_{2}\right), u_{2}\left(x_{1}, x_{2}\right)\right)\right]=\left(u_{1}\left(x_{1},-x_{2}\right),-u_{2}\left(x_{1},-x_{2}\right)\right) .
$$

The volumetric force term in Eq. (18) can be classified with respect to its symmetry properties. For electrode configuration $\mathrm{S} 3$, we have

$$
\mathbf{P}_{x_{2}}\left[\mathbf{f}_{\mathrm{S} 3}\right]=\mathbf{f}_{\mathrm{S} 3}
$$

while for the other two electrode configurations, the volumetric force term is not invariant under $\mathbf{P}_{x 2}$. Approximating the time derivative in Eq. (18) using a forward Euler scheme with a time increment $\Delta t$, we obtain

$$
\begin{aligned}
\frac{\delta \mathbf{u}(t+\Delta t)}{\Delta t} \approx & \frac{\delta \mathbf{u}(t)}{\Delta t}-\left(\mathbf{u}_{0} \cdot \nabla\right) \delta \mathbf{u}(t)-(\delta \mathbf{u}(t) \cdot \nabla) \mathbf{u}_{0} \\
& +\frac{1}{R e_{c h}}\left(\Delta \delta \mathbf{u}(t)-\nabla \delta p(t)+\mathbf{f}_{\mathbf{e}}\right) .
\end{aligned}
$$

Let us assume that at time $t$, the perturbation $\delta \mathbf{u}$ is invariant under $\mathbf{P}_{x_{2}}$. With the invariance of $\mathbf{u}_{0}$, we obtain the invariance of the pressure field $\delta p(t)$ under $\mathbf{P}_{x_{2}}$. To probe the invariance of $\delta \mathbf{u}$ at time $t+\Delta t$, we write

$$
\begin{aligned}
\mathbf{P}_{x_{2}}\left[\frac{\delta \mathbf{u}(t+\Delta t)}{\Delta t}\right] \approx & \mathbf{P}_{x_{2}}\left[\frac{\delta \mathbf{u}(t)}{\Delta t}-\left(\mathbf{u}_{0} \cdot \nabla\right) \delta \mathbf{u}(t)-(\delta \mathbf{u}(t) \cdot \nabla) \mathbf{u}_{0}+\frac{1}{R e_{c h}}\left(\Delta \delta \mathbf{u}(t)-\nabla \delta p(t)+\mathbf{f}_{\mathbf{e}}\right)\right] \\
= & \mathbf{P}_{x_{2}}\left[\frac{\delta \mathbf{u}(t)}{\Delta t}\right]-\mathbf{P}_{x_{2}}\left[\mathbf{u}_{0} \cdot \nabla\right] \mathbf{P}_{x_{2}}[\delta \mathbf{u}(t)]-\mathbf{P}_{x_{2}}[\delta \mathbf{u}(t) \cdot \nabla] \mathbf{P}_{x_{2}}\left[\mathbf{u}_{0}\right] \\
& +\frac{1}{R e_{c h}}\left(\mathbf{P}_{x_{2}}[\Delta \delta \mathbf{u}(t)]-\mathbf{P}_{x_{2}}[\nabla \delta p(t)]+\mathbf{P}_{x_{2}}\left[\mathbf{f}_{\mathbf{e}}\right]\right) .
\end{aligned}
$$

It can easily be shown that under the assumptions made, the terms involving the dot products between the velocity fields and the nabla operator are invariant under $\mathbf{P}_{x_{2}}$. Therefore,

$$
\begin{aligned}
\mathbf{P}_{x_{2}}\left[\frac{\delta \mathbf{u}(t+\Delta t)}{\Delta t}\right] \approx & \frac{\delta \mathbf{u}(t)}{\Delta t}-\left(\mathbf{u}_{0} \cdot \nabla\right) \delta \mathbf{u}(t)-(\delta \mathbf{u}(t) \cdot \nabla) \mathbf{u}_{0} \\
& +\frac{1}{R e_{c h}}\left(\Delta \delta \mathbf{u}(t)-\nabla \delta p(t)+\mathbf{P}_{x_{2}}\left[\mathbf{f}_{\mathbf{e}}\right]\right),
\end{aligned}
$$

which means that, given an invariant initial perturbation, the only effect that can remove the mirror-reflection symmetry of the perturbation flow field is the volumetric force $\mathbf{f}_{\mathbf{e}}$. If, on the other hand, $\mathbf{f}_{\mathbf{e}}$ is mirror symmetric as well, the flow field will remain in the space of mirror symmetric functions. However, we know that the von Kármán vortex street breaks the mirror symmetry with respect to $x_{2}$, and in that case there would be no way how the flow field can develop into a KVS. Of course, in reality (and also in numerical experiments) there are perturbations that break the mirror symmetry. This means that there are two different pathways of the system to develop a KVS: either by a volumetric force that breaks the mirror symmetry or by a symmetry-breaking perturbation of the invariant flow field $\mathbf{u}_{0}$.

Our linear stability analysis offers a qualitative explanation for the results displayed in Table II. The settling times obtained for the symmetry-breaking electrode configurations (S1 and S2) are significantly smaller than the settling time without an electric field. The reason is that in this case there are two different mechanisms for the invariant initial flow field to develop into a KVS. Otherwise the only pathway is via a perturbation to the flow that breaks the mirror-reflection symmetry.

\section{Drag coefficient}

We extract the drag coefficient of the fully developed LVSR from the simulation results according to Eq. (12). The corresponding time-averaged drag coefficients for various Reynolds numbers, electrokinetic manipulations, and the blockage ratios $\varepsilon=0.1,0.3$, and 0.5 are given in Figs. 10(a)-10(c), respectively. In general, the drag coefficient for a given confinement decreases when the Reynolds number increases. For the blockage ratio of $\varepsilon=0.1$, the drag coefficient approaches a more or less constant value at medium Reynolds numbers of the LVSR. For the higher confinements, a continuous decrease is observed. In terms of a given Reynolds number, the drag coefficient increases when the blockage ratio increases. This should be mainly related to the higher gap velocities due to the flow confinement which results in a drag force that corresponds to a flow with a higher Reynolds number. In terms of the influence of the electrokinetic manipulations, we find that case S1U does not influence the drag coefficient, while S2U and S2D always give higher and lower values, respectively. This can be explained by considering the equivalent electrostatic force acting on the cylinder. Electrokinetic manipulation $\mathrm{S} 1 \mathrm{U}$ results in a force solely in the vertical direction and therefore does not contribute to the drag coefficient which represents forces in the horizontal direction. For a given confinement, all curves have the same shape and it appears that an electrokinetic manipulation with a horizontal component merely shifts the curve on the $y$-axis. A simple scaling law $c_{D, 1}=\frac{c_{D, 0}}{1 \pm \frac{\Pi}{2} \cos (\varepsilon)}$, where $c_{D, 0}$ is the drag coefficient of the pure pressure-driven flow and $c_{D, 1}$ is the drag coefficient of the electrokinetic manipulation, can be used to shift the curves of S2D and S2U onto the pressure-driven one. 

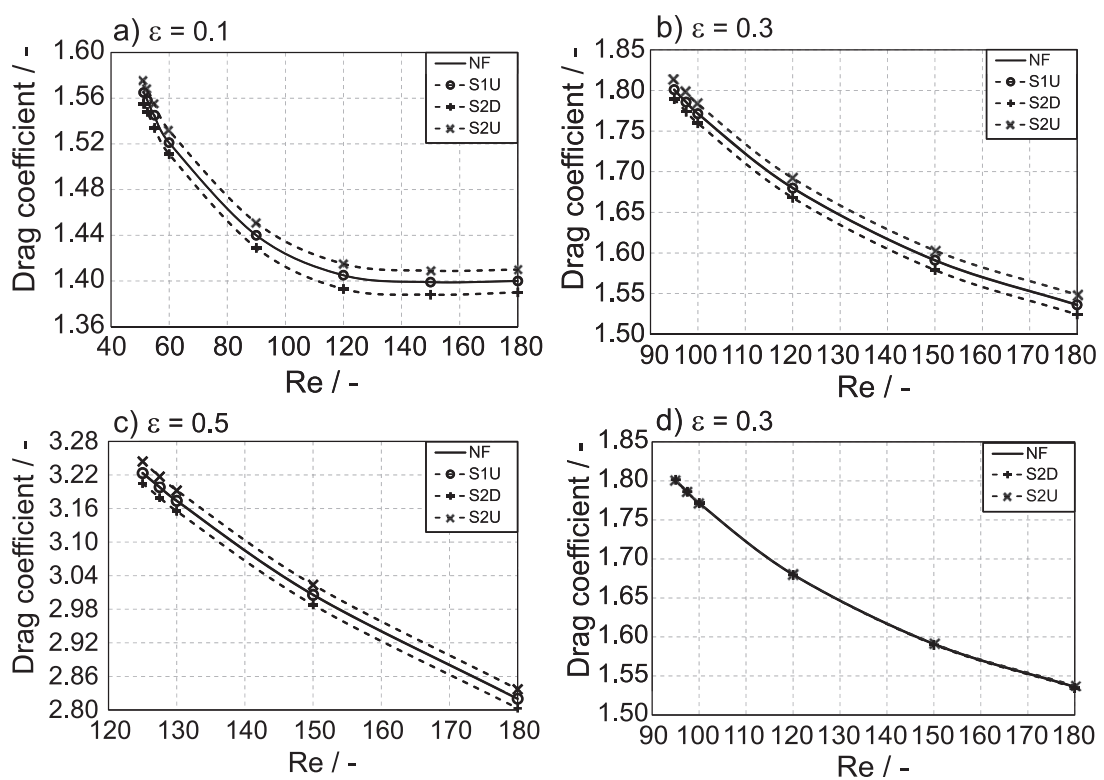

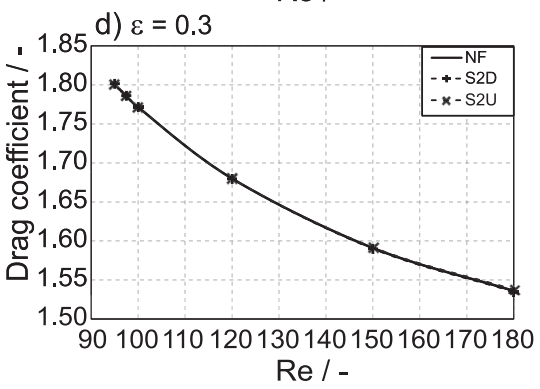

FIG. 10. Time-averaged drag coefficient versus Reynolds number for different electrokinetic manipulations and a blockage ratio of (a) $\varepsilon=0.1$; (b) $\varepsilon$ $=0.3$; (c) $\varepsilon=0.5$ as well as (d) Scaled drag coefficients for $\varepsilon=0.3$ where the data collapse on a single curve identical to that of the pure pressure driven flow. Lines are guides to the eyes.
The sign in the denominator depends on the equivalent electrostatic force direction where a component in the downstream and upstream direction increases and decreases the denominator, respectively. Figure 10(d) shows the time-averaged drag coefficient for the blockage ratio $\varepsilon=0.3$ where the data of the electrokinetic manipulations S2D and S2U are scaled so that they collapse on the curve of the pure pressure-driven flow with high accuracy.

To get a more detailed insight into the influence of the different electrokinetic manipulations, we compare the steady state oscillations of the drag coefficient at $R e=60, \varepsilon=0.1$, and $\Pi=0.014$ in Fig. 11 .

Part (a) shows that the drag coefficient of the pure pressure-driven flow corresponds to a harmonic oscillation around 1.521 with an amplitude of 0.0007 . As expected, the corresponding Strouhal number of the steady-state oscillation is 0.263 , the twofold value of the lift coefficient. This is understood since the period of the drag coefficient is the time between the shedding of upper and lower vortex. By contrast, the period of the lift coefficient is the time between the
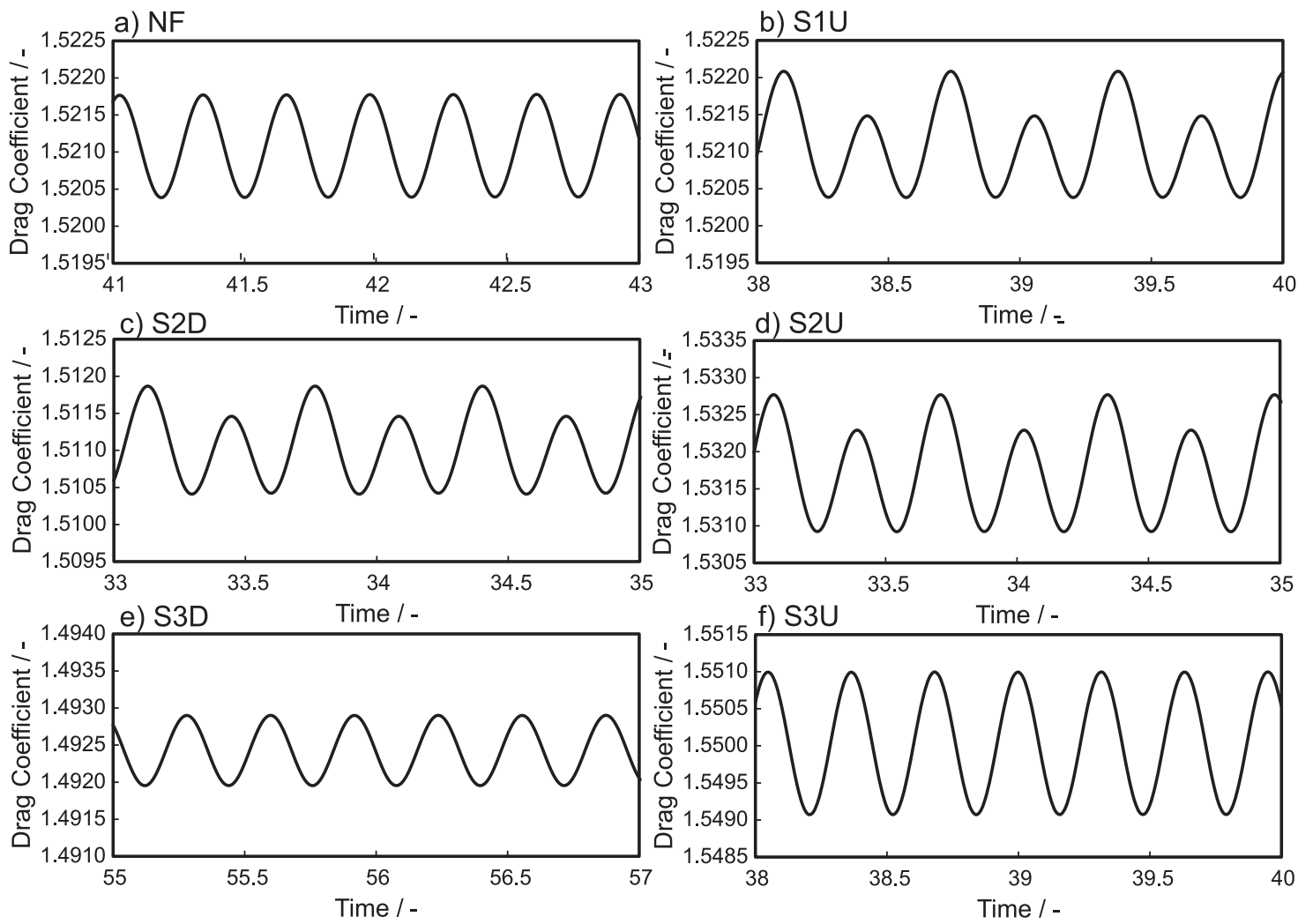

FIG. 11. Drag coefficient versus time for a flow with $\varepsilon=0.1, \Pi=0.014$, and $\operatorname{Re}=60$ : (a) NF (without electric field); (b) $\mathrm{S} 1 \mathrm{U}$ (aligned electrodes, field up); (c) S2D (displaced electrodes, field down); (d) S2U (displaced electrodes, field up); (e) S3D (field downstream); (f) S3U (field upstream). 
shedding of two vortices at the same location. Parts (b)-(f) depict the drag coefficient oscillation for the different electrokinetic manipulations. A striking observation is that configurations S1U, S2D, and S2U feature two characteristic frequencies. The additional frequency is the subharmonic $(n=1 / 2)$ of the drag coefficient frequency, i.e., the Strouhal number of the lift coefficient. This phenomenon is obviously related to the vertical equivalent electrostatic force component which triggers an oscillation where every second vortex at a location sheds at a higher drag coefficient value. Note that the FFT of the lift coefficient oscillation always gives a single characteristic frequency. We cannot identify a simple correlation between the integral drag coefficient parameters and the settling time. It is only obvious that an acceleration of the VKS development goes hand in hand with the appearance of the subharmonic frequency. Hence, they are obviously a sign or a result of the breaking of the (initial) flow symmetry due to the vertical equivalent electrostatic force.

Figure 12 shows the lift versus the drag coefficient over a period of the steady state vortex shedding for flows with $R e=60, \Pi=0.014, \varepsilon=0.1$, and different electrokinetic manipulations. This phase plot gives an enhanced visualization of the previous results since it displays both vertical and horizontal forces that act on the cylinder at the same time. Part (a) gives the phase plot of the pure pressure-driven flow. The observed double loop is a consequence of the symmetric alternate shedding, in which the lift coefficient has twice the period of the drag coefficient. The individual single loops are symmetric to each other with the symmetry line being located at $c_{L}=0$. When we compare the pure pressure-driven flow trajectories with those for flows with electrokinetic manipulations given in parts (b)-(f), there are obvious differences. Any equivalent electrostatic force component in the vertical direction shifts the junction of the double loop on the vertical axis and breaks its symmetry. A component in the up direction shifts the double loop in the up direction and enlarges the upper loop. A component in the down direction shifts the double loop in the down direction and enlarges the lower loop. That is, the manipulations S1U, S2U, and S2D that break the initial flow symmetry around the cylinder, and therefore reduce the settling time, also feature an asymmetric double loop where one loop is noticeably larger than its counterpart. It can also be seen right away that an equivalent electrostatic force in the horizontal direction has a direct influence on the amplitude of the lift coefficient oscillation.

\section{Vorticity in the wake}

Another possibility to evaluate the influence of the electrokinetic manipulation is to investigate the vorticity $\omega=\nabla \times \mathbf{u}$ at a certain position in the wake of the cylinder. Here, we sample the vorticity at the channel centerline at a distance of $h$ to the cylinder center; a location which is chosen such that the detached vortices have to pass it.

Figure 13(a) shows the temporal evolution of the vorticity in the cylinder wake for a pure pressure-driven flow with $R e=60$ and $\varepsilon=0.1$. Similar to the lift coefficient, the vorticity develops over time and becomes steady after a settling time of around $t=23.3$. In terms of electrokinetic manipulation, the influence on the temporal evolution (settling time) is similar to that of the lift coefficient and we abstain from showing these results. Nevertheless, a FFT analysis of the steady state vorticity oscillation, given in Fig. 13(b), reveals some interesting aspects. In contrast to the lift coefficient, the Fourier spectrum of the vorticity consists of four dominant frequencies. Generally, we observe that the higher the Reynolds number, the higher the number of dominant frequencies and
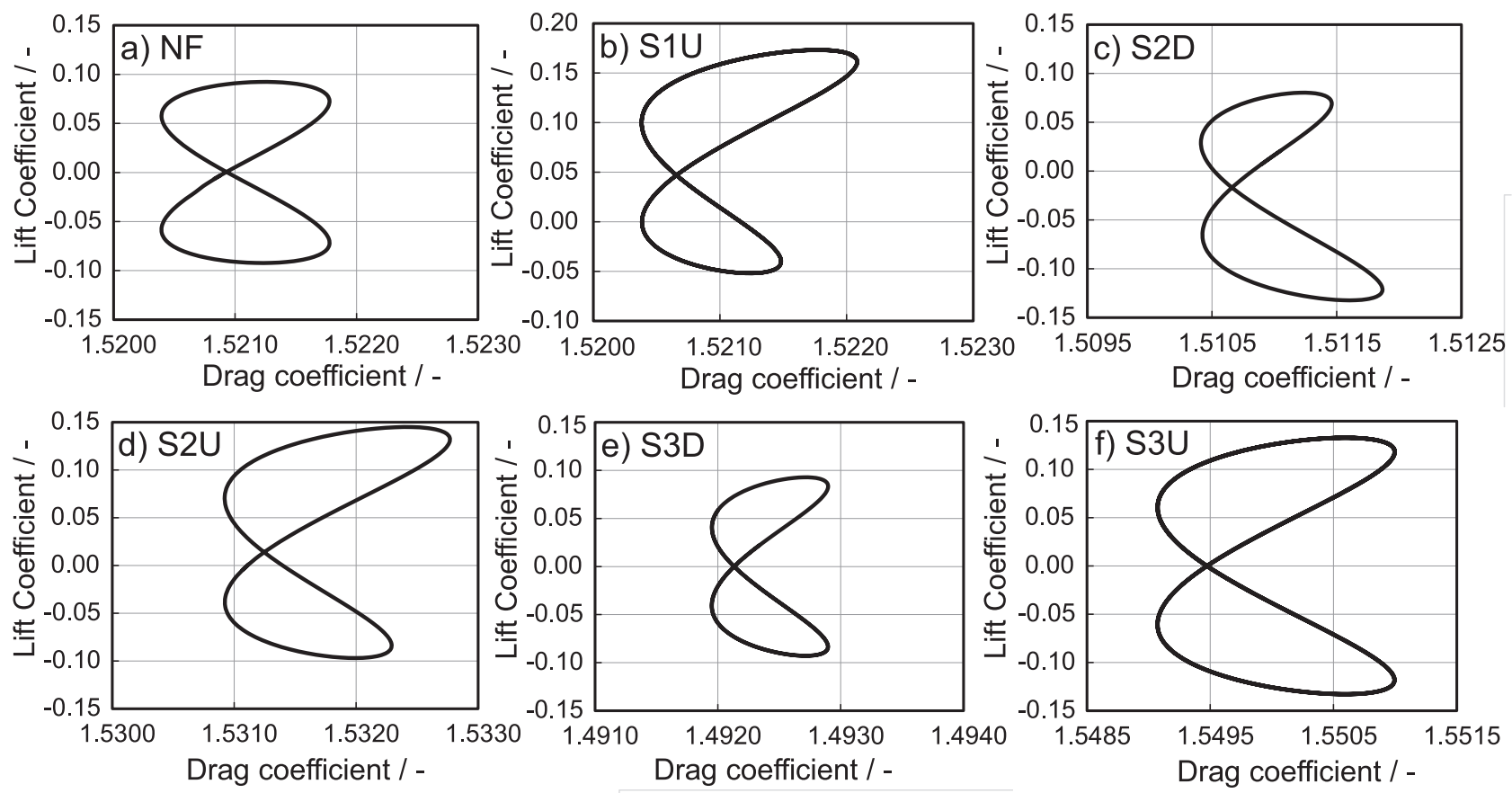

FIG. 12. Phase plot of lift and drag coefficient for $\varepsilon=0.1$ and $R e=60$ : (a) NF (without electric field); (b) S1U (aligned electrodes, field up); (c) S2D (displaced electrodes, field down); (d) S2U (displaced electrodes, field up); (e) S3D (field downstream); (f) S3U (field upstream). 

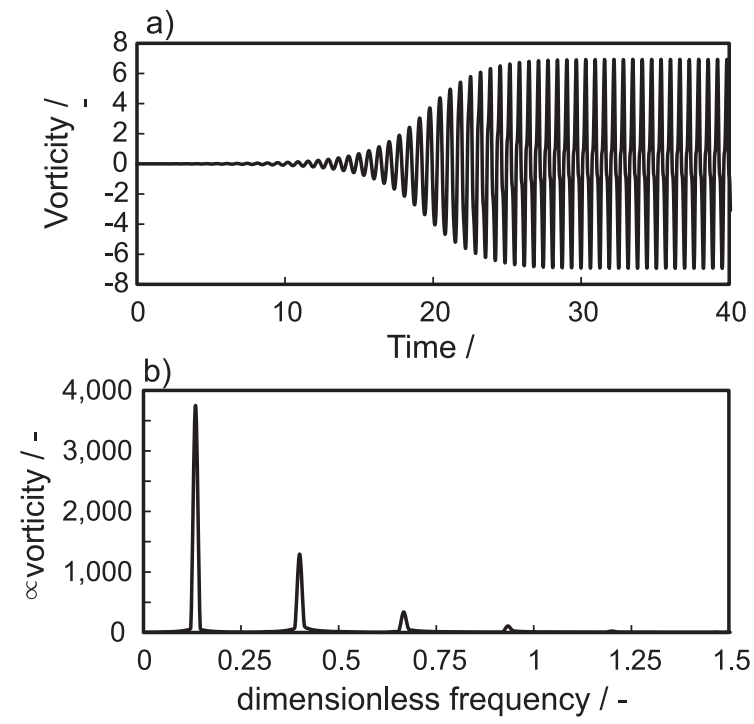

FIG. 13. Vorticity in the cylinder wake for pure pressure-driven flow with $\varepsilon=0.1$ and $R e=60$ : (a) temporal evolution and (b) frequency spectrum of the steady state oscillation.

the higher their values. The lowest frequency always corresponds to the Strouhal number $(n=1)$ of the lift coefficient. The other frequencies are always odd superharmonics of the Strouhal number with multiples of $n=3,5, \ldots$ The confinement lowers the number of the dominant frequencies and also decreases their values. Further comparison reveals that the dominant frequencies of the vorticity spectrum correspond to those of the horizontal velocity component. By contrast, the spectrum of the vertical velocity component comprises even multiples of the Strouhal number; that is $n=2,4,6$. Note that if we consider the drag coefficient frequency as harmonic mode $(n=1)$, we again arrive at superharmonics with odd multiples of $n=3,5,7$.

The appearance of further characteristic frequencies can be a sign for a flow transition (bifurcation). A flow may become chaotic (turbulent) when it undergoes a number of transitions, and different scenarios are known that result in chaotic flows which depend on geometry, initial conditions, and other specific flow features. ${ }^{22}$ An example for frequency doubling is the backward-facing step where the analysis of the (periodic) flow reveals the appearance of further superharmonic frequencies $(n=2,3,4, \ldots)$ with increasing Reynolds number. Eventually, an infinite number of superharmonic frequencies is observed and chaos sets in Ref. 23.

For the cylinder wake, a sequence of period doubling bifurcations, the so-called Feigenbaum scenario, ${ }^{24}$ can be observed and is considered as a sign for the transition of a $2 \mathrm{D}$ to a $3 \mathrm{D}$ flow. $^{7,25}$ In measured wake velocity spectra, not only the $n=1 / 2$ but also the $n=1 / 3$ subharmonic mode can be found in certain ranges of the Reynolds numbers. ${ }^{7}$ By contrast, Zhang et al. measured additional superharmonic modes in the power spectrum with $n=2$ at $R e=177$ and $n=2,3$ at $R e=217$ which are assigned to three different 3D vortex shedding modes. ${ }^{26}$

There is a another scenario that is related to the occurrence of multiple dominant frequencies. At higher Reynolds numbers of the LVSR of a confined flow, there is also the possibility of the so-called beating phenomenon when vortices shed from the channel walls. ${ }^{11,13,14}$ We observe this phenomenon in our simulation results as well but the superharmonic frequencies can even be found at the lowest blockage ratio and Reynolds numbers. Hence, we perform further limited simulations at $R e=60$ without confinement of the cylinder flow. This is achieved by a ratio of the cylinder diameter to the height of the simulation domain of $1 / 60$ in conjunction with a no-stress condition. In contrast to the confined case, we recover the $n=2$ frequency (drag coefficient) in the vertical velocity spectrum and the $n=1$ frequency (lift coefficient) in the horizontal velocity spectrum. Hence, we are able to identify all (super) harmonic modes of the Strouhal number $n=1,3,2,5,4$ (in order of signal strength) in the vorticity spectrum. On the one hand, this clarifies that these additional frequencies are not related to wall vortex shedding; they may be a sign for flow transition. On the other hand, it demonstrates that the wall confinement suppresses selected superharmonic frequencies in the velocity spectra. In detail, the walls suppress the even multiples in the vertical velocity spectrum and the odd multiples in the horizontal velocity spectrum.

We also inspect the influence of the Reynolds number, blockage ratio, and the electrokinetic manipulation on the vorticity amplitude (results not shown). For a constant blockage ratio, the amplitude increases with increasing Reynolds number. For a constant Reynolds number, the damping effect of the walls is distinct. For example, the vorticity amplitude at $R e=150$ decreases from around 35 to 4 when the blockage ratio increases from 0.1 to 0.5 . In terms of electrokinetic manipulations, similar relationships as shown for the lift coefficient in Fig. 9 are observed.

\section{CONCLUDING REMARKS}

The present research is concerned with the numerical simulation of the pressure-driven flow around a confined cylinder subjected to a DC electric field. In this setup, two distinctions to the classic von Kármán vortex street can be noted. One is the confinement of the flow field that differs from its unbounded counterpart in terms of the shear in the incoming velocity profile, shear induced in the vicinity of the boundaries, and with the impermeability of the channel walls for vortices. The other is the DC electric field that induces a very small electrokinetic velocity at the cylinder surface in contrast to the flow situation arising from the no-slip velocity in the conventional case. A comprehensive numerical study of these electrokinetically manipulated flows is conducted in the Laminar Vortex Shedding Regime. In detail, we first investigate the influence of the blockage ratio on pressure-driven flows. The simulation results show that the confinement increases the critical Reynolds number and the Strouhal number of the vortex shedding. A scale analysis reveals that the increase in the Strouhal number is related to the increase in the gap velocity due to the confinement. The selected simulation results are compared to the respective literature, and the very good agreement validates the numerical schemes that we use in this research.

In the next step, various electrokinetic manipulations are investigated where we use electrode arrangements that span 
up different electric fields. The electric field induces electrokinetic velocities at the cylinder surface which are very much smaller than that of the channel flow. Nevertheless, the small electrokinetic flow can have a considerable impact on the flow topology around the cylinder. The use of an equivalent electrostatic force, resulting from integration along the cylinder surface, facilitates the result interpretation. We observe that a force with a component in the vertical direction to the channel breaks the (initial) axial flow mirror reflection symmetry of the Laminar Steady Regime. This generally results in a considerable reduction of the settling time; i.e., the time that it takes until a steady vortex detachment is established. A qualitative linear stability analysis reveals that this can be explained by the fact that a mirror-symmetry breaking force opens an additional pathway for the flow to develop into a symmetrybreaking configuration. Close to the critical Reynolds number, a horizontal (symmetry conserving) equivalent electrostatic force component in the upstream direction triggers the opposite effect, i.e., a damping of the vortex shedding. A more general, and logic observation is that the vertical force component influences the characteristics of the lift coefficient, while the horizontal force component influences the drag coefficient. The Strouhal number of the vortex shedding (lift coefficient) is not influenced by the electrokinetic manipulation with a DC field.

However, a vertical equivalent electrostatic force component triggers the appearance of a subharmonic oscillation of the drag coefficient with the frequency of the lift coefficient. We also find a considerable influence of the electrokinetic manipulation on the vorticity of the wake flow at low Reynolds numbers of the Laminar Vortex Shedding Regime. The vorticity spectra do not only comprise the vortex shedding frequency but also various superharmonic frequencies. Finally, the evaluations of lift and drag coefficient and vorticity show that the same small perturbation can, depending on the Reynolds number and field direction, have opposite (acceleration and damping) influences on the properties of the vortex street which just underlines the high complexity of such manipulated flows.

In future work, a comprehensive stability analysis by computing the eigenvalues of the problem should be pursued. The question of the superharmonic modes in the (velocity) vorticity spectrum deserves a closer look to clarify whether they are related to the transition of flow to a more complex state. Finally, another logical extension of this work would be the manipulation with an AC electric field of low frequency to identify lock-in states of the vortex shedding.

\section{ACKNOWLEDGMENTS}

M.S. would like to thank the Deutsche Akademische Austausch Dienst (DAAD) for funding his stay at Queen's University. D.P.J.B. is grateful to the Alexander von
Humboldt Foundation for supporting his research at TU Darmstadt with a Research Fellowship.

${ }^{1}$ S. Ghosal, "Electrokinetic flow and dispersion in capillary electrophoresis," Annu. Rev. Fluid Mech. 38, 309-338 (2006).

2 Á. V. Delgado, F. González-Caballero, R. Hunter, L. Koopal, and J. Lyklema, "Measurement and interpretation of electrokinetic phenomena," J. Colloid Interface Sci. 309, 194-224 (2007).

${ }^{3}$ C. Zhao and C. Yang, "Advances in electrokinetics and their applications in micro/nano fluidics," Microfluid. Nanofluid. 13, 179-203 (2012).

${ }^{4}$ E. Berger and R. Wille, "Periodic flow phenomena," Annu. Rev. Fluid Mech. 4, 313-340 (1972).

${ }^{5}$ P. A. Irwin, "Vortices and tall buildings: A recipe for resonance," Phys. Today 63(9), 68-69 (2010).

${ }^{6} \mathrm{Y}$. Chen, "Flow-induced vibration and noise in tube-bank heat exchangers due to von Karman streets," J. Eng. Ind. 90, 134-146 (1968).

${ }^{7}$ C. H. Williamson, "Vortex dynamics in the cylinder wake," Annu. Rev. Fluid Mech. 28, 477-539 (1996).

${ }^{8}$ P. Roushan and X. Wu, "Universal wake structures of Kármán vortex streets in two-dimensional flows," Phys. Fluids 17, 073601 (2005).

${ }^{9}$ L. Zovatto and G. Pedrizzetti, "Flow about a circular cylinder between parallel walls," J. Fluid Mech. 440, 1-25 (2001).

${ }^{10}$ J.-H. Chen, W. Pritchard, and S. Tavener, "Bifurcation for flow past a cylinder between parallel planes," J. Fluid Mech. 284, 23-41 (1995).

${ }^{11}$ M. Sahin and R. G. Owens, "A numerical investigation of wall effects up to high blockage ratios on two-dimensional flow past a confined circular cylinder," Phys. Fluids 16, 1305-1320 (2004).

${ }^{12}$ B. Kumar and S. Mittal, "Effect of blockage on critical parameters for flow past a circular cylinder," Int. J. Numer. Methods Fluids 50, 987-1001 (2006).

${ }^{13}$ F. Rehimi, F. Aloui, S. B. Nasrallah, L. Doubliez, and J. Legrand, "Experimental investigation of a confined flow downstream of a circular cylinder centred between two parallel walls," J. Fluids Struct. 24, 855-882 (2008).

${ }^{14}$ M. D. Griffith, J. Leontini, M. C. Thompson, and K. Hourigan, "Vortex shedding and three-dimensional behaviour of flow past a cylinder confined in a channel," J. Fluids Struct. 27, 855-860 (2011).

${ }^{15} \mathrm{I}$. Meisel and P. Ehrhard, "Electrically-excited (electroosmotic) flows in microchannels for mixing applications," Eur. J. Mech. B: Fluids 25, 491-504 (2006).

${ }^{16}$ D. Barz, H. Zadeh, and P. Ehrhard, "Measurements and simulations of timedependent flow fields within an electrokinetic micromixer," J. Fluid Mech. 676, 265-293 (2011).

${ }^{17}$ P. Amestoy, I. Duff, and J.-Y. L'excellent, "Multifrontal parallel distributed symmetric and unsymmetric solvers," Comput. Methods Appl. Mech. Eng. 184, 501-520 (2000).

${ }^{18} \mathrm{O}$. Schenk and K. Gärtner, "Solving unsymmetric sparse systems of linear equations with PARDISO," Future Gener. Comput. Syst. 20, 475-487 (2004).

${ }^{19}$ U. M. Ascher and L. R. Petzold, Computer Methods for Ordinary Differential Equations and Differential-Algebraic Equations (SIAM, 1998), Vol. 61.

${ }^{20} \mathrm{C}$. Williamson, "Defining a universal and continuous Strouhal-Reynolds number relationship for the laminar vortex shedding of a circular cylinder," Phys. Fluids 31, 2742-2744 (1988).

${ }^{21}$ E. Dowell and J. Jaworski, "Tutorial on scaling analysis of Navier-Stokes equations: Linear and non-linear dynamics," J. Aeroelasticity Struct. Dyn. 2, 79-84 (2011).

${ }^{22}$ A. Guzmán and C. Amon, "Transition to chaos in converging-diverging channel flows: Ruelle-Takens-Newhouse scenario," Phys. Fluids 6, 1994-2002 (1994).

${ }^{23}$ H. Rani and T. W. Sheu, "Nonlinear dynamics in a backward-facing step flow," Phys. Fluids 18, 084101 (2006).

${ }^{24}$ M. J. Feigenbaum, "Universal behavior in nonlinear systems," Phys. D 7, 16-39 (1983).

${ }^{25}$ A. Tomboulides, G. Triantafyllou, and G. Karniadakis, "A new mechanism of period doubling in free shear flows," Phys. Fluids A 4, 1329-1332 (1992).

${ }^{26}$ H.-Q. Zhang, U. Fey, B. R. Noack, M. König, and H. Eckelmann, "On the transition of the cylinder wake," Phys. Fluids 7, 779-794 (1995). 